Journal of Agricultural Sciences
(Tarim Bilimleri Dergisi)

\title{
Resistance Response of Drought and Heat Tolerant Spring Wheat Lines against the Cereal Cyst Nematode, Heterodera filipjevi
}

\author{
Osameh ATIYA $^{\mathrm{a}^{*}} \mathbb{D}$, Abdelfattah DABABAT ${ }^{\mathrm{b}} \mathbb{D}$, Gul ERGINBAS-ORAKCI $^{\mathrm{b}} \mathbb{( D}$, Sukhwinder SINGH $^{\mathrm{C}} \mathbb{D}$, \\ Halil TOKTAY ${ }^{a}$ (D)

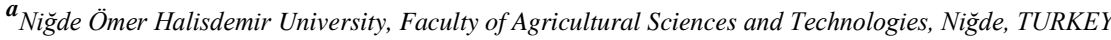 \\ $\boldsymbol{b}$ International Maize and Wheat Improvement Centre - Turkey office P.K. 39 Emek 06511 Ankara, TURKEY

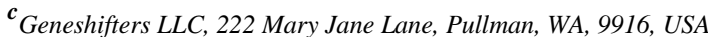

ARTICLE INFO

Research Article

Corresponding Author: Osameh ATIYA, E-mail: osamehatiya@yahoo.com

Received: 5 November 2020 / Revised: 26 May 2021 / Accepted: 27 May 2021 / Online: 25 March 2022

\section{ABSTRACT}

Wheat (Triticum aestivum L.) is extremely affected by several abiotic and biotic stress factors. Drought and/or heat alongside the parasitism of cereal cyst nematodes of the Heterodera genera can have a combined destructive impact on wheat. Solely, the cereal cyst nematode species Heterodera filipjevi can cause wheat yield losses of up to $50 \%$. Several control measures have been implemented, yet the most economical and convenient control strategy is the use of resistant hosts. Therefore, the main aim of this study was to evaluate the resistant response of 257 spring wheat lines obtained from the International Maize and Wheat Improvement Centre to Heterodera filipjevi that might contain novel sources of resistance and be added as genetic resources for future breeding programs. Also, provide a base for future research to understand the relationship between nematode resistances and drought and heat tolerance. The results indicated that 11 wheat lines $(4 \%)$ and 36 wheat lines $(14 \%)$ were resistant and moderately resistant, respectively. High frequency of susceptible and highly susceptible lines and low frequency of resistant lines within this set was also recorded. The linear regression analysis between the number of cysts formed and the resistance response grouping showed a strong, positive, linear correlation. Log-linear regression analysis showed that there is a weak positive correlation between the yield of heat tolerant wheat lines and their resistance to the cyst nematodes as these lines showed tolerance, while there was a weak negative correlation of formed cyst nematodes on the yield of drought tolerant lines. This study was able to add new genetic sources of resistance to Heterodera filipjevi for upcoming breeding programs.

Keywords: Biotic stress, Breeding, Cereal crops, Plant-parasitic nematodes, Resistant source

(C) Ankara University, Faculty of Agriculture

\section{Introduction}

Bread wheat (Triticum aestivum L.) is a vital crop for many countries, contributing nearly to one-third of the total global food grain production (FAOSTAT 2020). Abiotic and biotic stress factors can interact together to adversely affect wheat yield and production worldwide (Lichtenthaler 1996; Afzal et al. 2015). In the wheat gene pool, there is an adequate genetic variation that can ensure continuous improvement of wheat adaptation to these stress factors (Lawlor \& Cornic 2002; Trethowan \& MujeebKazi 2008).

Climate change is a challenge facing humanity and its effects have been harmful to the agricultural industry. It is projected that countries near the equator will have a reduction in food production (Droogers \& Aerts 2005). The International Water Management Institute (IWMI) study forecasts that wheat production in South Asia will decline by $50 \%$ by 2050 (De Fraiture et al. 2007). Studies indicated that increasing temperatures have negative effects on wheat yields in numerous regions of the world (Parry et al. 2004; Asseng et al. 2015; Zhao et al. 2017). It is going to be a challenge to increase or at least maintain the world's production of wheat to provide future generations with food needed to satisfy the demands of the increasing population. Also, it is a current and future challenge to find ways to reduce the impact of stress factors that decreases the yield of wheat such as plant-parasites (i.e., plant-parasitic nematodes) along with environmental factors (i.e., drought and heat).

Plant-parasitic nematodes that are the cause of agriculture production reduction, are regarded to be alarming. Despite their widespread compared to other pests, plant-parasitic nematodes are commonly very dangerous, stealthy, and costly to be managed and there is not enough detailed information or data on their economic impact (Webster 1987; Vaish 2017). Handoo (1998) valued the losses of international crop production due to nematode infection was around US\$ 80 billion and McCarter (2009) estimated that the global economic loss due to nematode infection to be US\$ 118 billion per year. The widespread of plantparasitic nematodes on a majority of the vital crops, especially cereal cyst nematodes (CCNs) on wheat and their effect that 
dramatically reduces crop yields has caught the awareness of governments and international organizations to find methods of management.

Nicol et al. (2011) reported that environmental conditions influence losses that are caused by CCNs and may exceed $90 \%$. In association with other biotic and abiotic factors such as fungal pathogens, water stress, and heat, CCNs can have a synergistic destructive impact (Nicol et al. 2006). The species of CCN avenae complex H. avenae, H. filipjevi and H. latipons (Rivoal \& Cook 1993; Nicol \& Rivoal 2008; Akar et al. 2009; Dababat et al. 2015; Seid et al. 2021) are considered the most economically important species in West Asia, North Africa, and the Mediterranean (Nicol et al. 2011; Dababat et al. 2021). H. filipjevi can be found generally in China, Germany, India, Iran, Norway, Poland, Russia, Spain, Syria, Sweden, Tajikistan, Turkey, and the USA (Nicol et al. 2006). In Turkey, H. filipjevi causes yield losses of up to $50 \%$ in winter wheat under rainfed conditions (Nicol et al. 2006; Imren et al. 2019). While Hajihasani et al. (2010) reported that in Iran H. filipjevi causes approximately 48\% yield losses in winter wheat under rainfed conditions. Additionally, Karimipour Fard et al. (2018) reported that $H$. filipjevi in field conditions significantly had a negative effect on grain yield (23\% reduction) and growth parameters (plant height, number of tillers, root dry weight, root height and aerial shoot dry weight) in all of the tested three cultivars.

Cereal cyst nematodes management methods have been mainly attained by rotation with non-host crops, such as legumes and moderately resistant cultivars. Due to the multi-year survival nature of the cyst that protects the eggs, a crop rotation period of at least 2 years is needed to maintain population densities below the economic damage threshold (Bridge \& Starr 2007). This might be considered a non-feasible and non-profitable way of management due to its time and cost in cultivating practices during crop rotation. Host resistance is a desirable alternative because it is less expensive, easy to be used once identified, and it has no environmental toxicity like nematicides, despite the successful use of nematicides to control nematodes (Williamson \& Kumar 2006, Dababat \& Fourie 2018). Dababat et al. (2014) stated that globally, the evolution of cultivars with genetic resistance plus genetic tolerance has been accomplished. Screens (resistant tests) have been established for almost every crop to present phenotypic data for their particular breeding program. These screens aim to find and identify new sources of resistance and also the identification of resistant progeny in segregating populations. Miniaturized screening test either by using the test tube method is considered to show the greatest accuracy regarding phenotypic reaction distinction (Blok et al. 2018).

The study aims to screen and evaluate a set of drought and heat tolerant international spring wheat lines for the resistance response to $H$. filipjevi. It is believed that this set is a unique set for $\mathrm{CCN}$ resistance because of their drought and heat tolerant nature, which is believed to have a relation with $\mathrm{CCN}$ resistance in general but needs other in-depth studies to establish this theory and our study is considered to be a starting point. It is also believed that this study would provide new resistant spring wheat lines against $H$. filipjevi, which might contain novel sources of resistance and be added as genetic resources for future breeding programs. Another primary expectation of the research is to provide a base for future research to try to understand the relationship between nematode resistances and drought and heat tolerance.

\section{Material and Methods}

\subsection{Plant Materials}

A set of 257 spring wheat lines that originated from various countries were obtained from the International Maize and Wheat Improvement Center (CIMMYT) in Mexico (Supplementary Table 1). This set has been screened and genotyped for drought and heat tolerance traits by CIMMYT-Mexico. The set was tested for cereal cyst nematode resistance response at the Transitional Zone Agricultural Research Institute (TZARI) in Eskisehir, Turkey (39 46' 1.2612" N, 30 24' 10.8282" E) and has been repeated in two independent experiments. Four well-known check lines for their resistance response to $H$. filipjevi were used as reference: 2 susceptible cultivars (Bezostaya and Kutluk-94) and 2 moderate resistant cultivars (Katea-1 and Sonmez-2001).

\subsection{Nematode inoculum: collection, hatching, and identification}

Soil samples were collected from a wheat field historically known to be infested with $H$. filipjevi in Çiçekdağ district in the province of Kirşehir, Turkey (39 63' 80" N; 34 46' 72" E). Cysts were extracted using Cobb's decanting and sieving method (Cobb, 1918). Cysts were handpicked from the organic matter residue under a dissecting microscope (Olympus SZ61). Then the cysts were surface sterilized with $\mathrm{NaOCl}(0.5 \%)$ for about 10 minutes and rinsed several times with distilled $\mathrm{H}_{2} \mathrm{O}$ before being transferred onto a fine mesh $(45 \mu \mathrm{m})$ placed in a glass petri dish and stored at $4{ }^{\circ} \mathrm{C}$ to enhance hatching. Freshly hatched secondstage juveniles (J2s) were used as an inoculum source. Species identification was previously validated by CIMMYT-Turkey using molecular methods from random individual cysts and identified as H. filipjevi (Pariyar et al. 2016a). To reinsure that there were no other species, morphological identification was done by using a light microscope (LEICA DM5500 B) along with imaging software Leica Application Suite (LAS V4.12) in the labs of the Faculty of Agricultural Sciences and Technology of Nigde Omer Halisdemir University ( $37^{\circ} 56^{\prime} 36.2^{\prime \prime}$ N; $34^{\circ} 37^{\prime} 42.4^{\prime \prime}$ E), as the fenestra of the cyst was the main aspect of species determination. The morphological measurements and characteristics were compared to previously published data of Siddiqi (2000); Handoo (2002); Subbotin \& Baldwin (2010). 


\subsection{Assessment of wheat lines}

Three representative spikes of a single wheat line were selected and threshed. About 15-20 similar and healthy seeds per line were selected and germinated on a moist filter paper in a Petri dish for 3 days at $22{ }^{\circ} \mathrm{C}$ and $70-80 \%$ of relative humidity. A single germinated seed of each line with identical sized radicles was selected and transplanted into RLC4-pine tubes $(25 \mathrm{~mm} \times 160 \mathrm{~mm}$ Ray Leach Cone-tainer ${ }^{\mathrm{TM}}$; Stuewe \& Sons, Inc., USA) containing $100 \mathrm{~g}$ of a sterilized growing mixture containing sand, organic matter, and field soil (70:29:1, v/v/v). The tubes were placed in a 200-cell tray (RL200; Ray Leach Cone-tainer ${ }^{\mathrm{TM}}$; Stuewe \& Sons, Inc., USA) with 3 replications per line arranged in a randomized block design. One day after transplanting, each tube was inoculated with 250 freshly hatched J2s suspended in $1 \mathrm{ml}$ of water and injected into 3 holes of $2 \mathrm{~cm}$ depth made by a thin plastic rod around the stem base. Plants were kept under controlled conditions $\left(25^{\circ} \mathrm{C}, 70 \% \mathrm{RH}\right.$ and a photoperiod of $\left.16 \mathrm{~h}\right)$ at TZARI. Fourteen weeks after nematode inoculation, plants were uprooted and cysts were extracted from both roots and soil of each plant as per Dababat et al. (2014). The resistance response of the screened wheat lines was determined and classified into five groups based on the mean number of cysts and females per plant (Dababat et al. 2016). The following grouping was used: 1 ) $\mathrm{R}=$ Resistant (fewer cysts and females/plant than the moderately resistant checks). 2) MR = Moderately resistant (as few cysts and females/plant as the moderately resistant checks). 3) MS = Moderately susceptible (significantly more cysts and females/plant than in the moderately resistant check, but not as many as in the susceptible checks). 4) $\mathrm{S}=$ Susceptible (as many cysts and females/plant as in the susceptible check and the number of cysts per root system considered damaging). 5) HS = Highly susceptible (more cysts and females/plant than in the susceptible check).

\subsection{Statistical analysis}

Descriptive statistical parameters (mean, standard error (SE) and standard deviation (SD)) of the number of cysts and females per line were calculated and compared with the check cultivars for their resistant response evaluation. Regression analysis was conducted to assess the correlation between the mean number of cysts and females and the sorting of the resistance response grouping and to calculate the best fitting equation, a polynomial regression analysis was used. Log-linear regression analysis was conducted to assess the correlation between the yield of drought and heat tolerant wheat lines and the mean number of formed cysts.

\section{Results and Discussion}

\subsection{Assessment of wheat lines}

The results of the screening evaluation of the 257 spring wheat lines showed that 11 lines (4.28\%) were resistant, 36 lines (14\%) were moderately resistant, 72 lines $(28.02 \%)$ were moderately susceptible, 79 lines $(30.74 \%)$ were susceptible and 59 lines $(22.96 \%)$ were highly susceptible, as shown in Figure 1 which also indicates a high frequency of susceptible and highly susceptible lines and low frequency of resistant lines within this set of drought and heat tolerant lines and represents a histogram distribution of the mean number of cysts formed on the root systems and estimated kernel density plot of the mean number of cysts formed per plant.

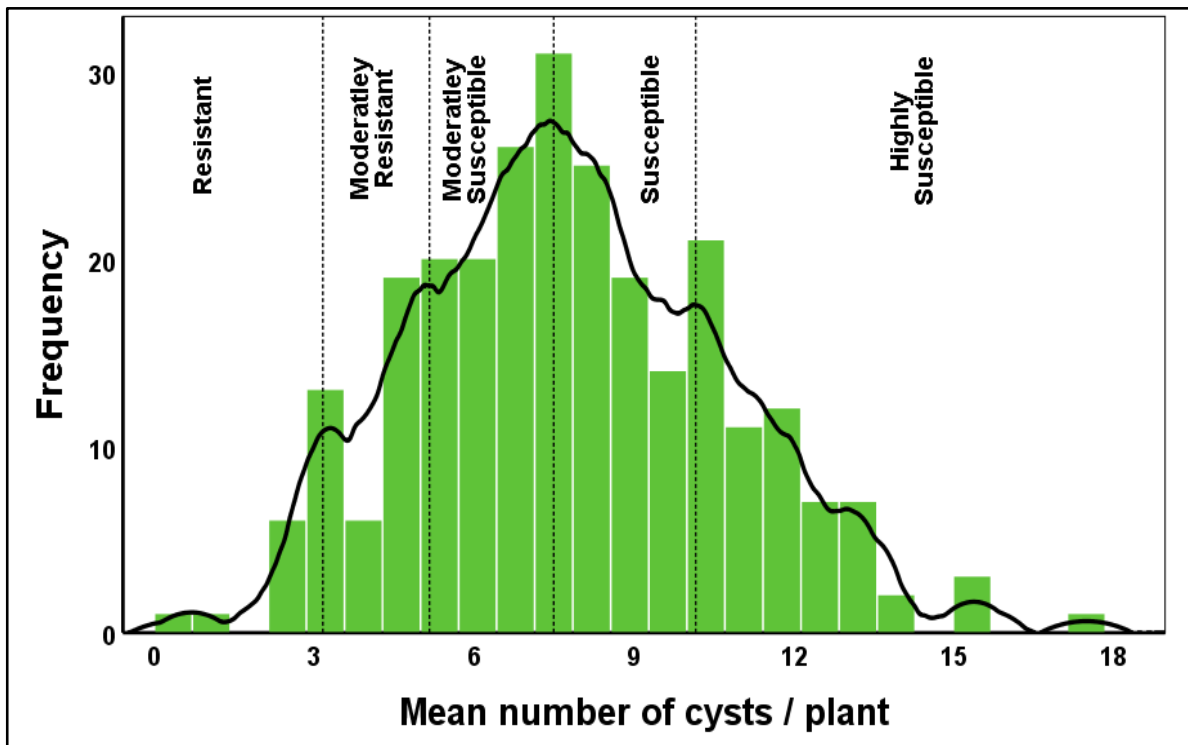

Figure 1- Histogram distribution and estimated kernel density plot of the mean number of cysts formed per plant, Frequency represents the number of wheat lines that cysts have formed on their root system between 0 - 18 


\subsection{Wheat lines resistant response grouping}

To further assess the correlation between the mean number of cysts and females and the resistance response grouping of the wheat lines, linear regression analysis was used $\left(\mathrm{P}<0.05, \mathrm{R}^{2}=0.958\right)$. The data points to a strong, positive, linear correlation between the number of cysts and females formed and the resistance response grouping (Figure 2), which shows that the increase of the number of cysts formed leads to the categorization of the wheat lines from resistant to highly susceptible.

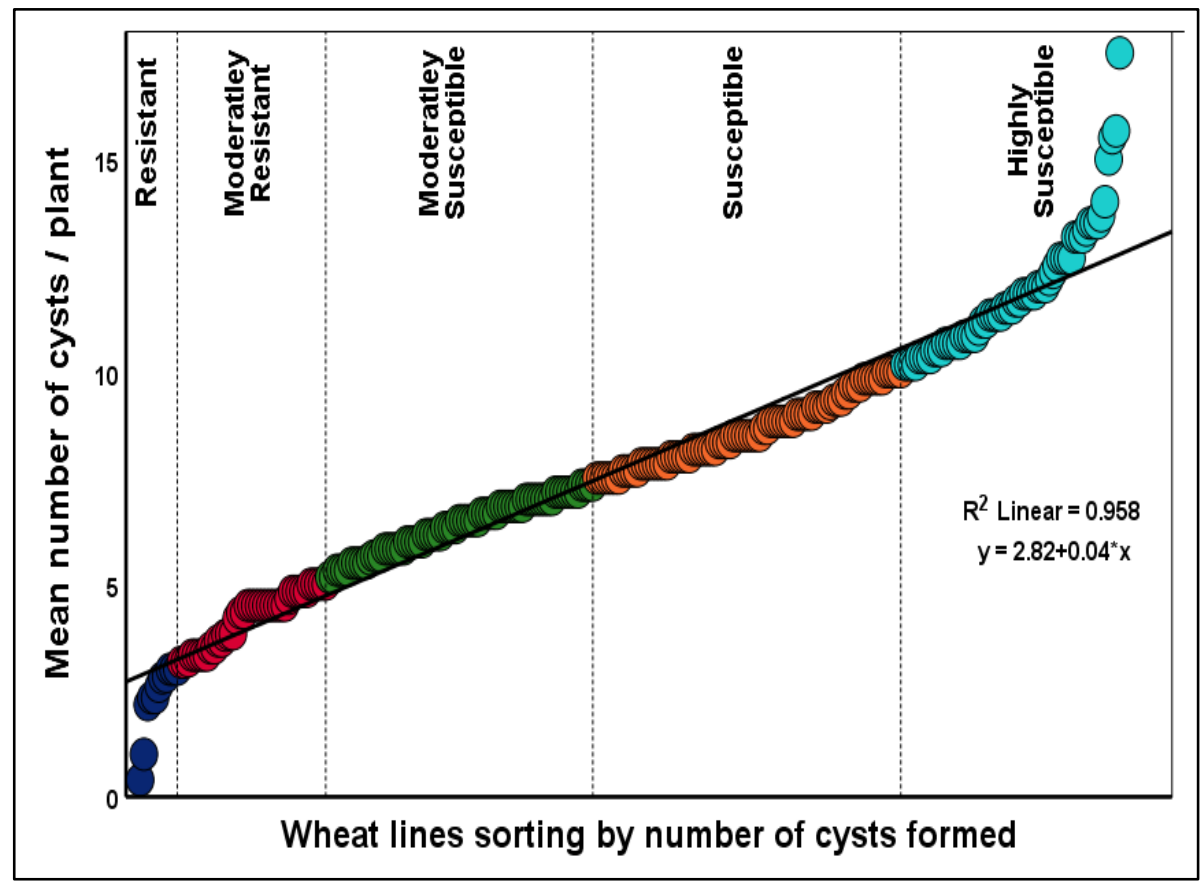

Figure 2- Linear regression of the mean number of cysts formed per plant and wheat lines sorting. Values are a mean of 2 trials, each with 3 replications. $(\mathbf{P}<0.05)$

\subsection{Source of resistance}

After examining and comparing the parent materials of the wheat lines that have shown resistant and moderately resistant responses to all the spring wheat lines within this set, it has been found that there is no coherent pattern or indication that one parent tends to pass down resistance traits to their pedigree over another. As the parent material of the resistant and moderately resistant lines can be also found in the moderately susceptible, susceptible, and highly susceptible lines. So, the source of resistance of this set is due to different factors other than the dominant traits of a certain parent in resistant and moderately resistant lines (data not presented). Further analysis is required to be conducted to get a better idea of what the resistant sources are.

\subsection{Effect of cyst nematodes on yield}

In order to assess the effect of drought and heat on the relation between wheat yield and the mean number of formed cysts, Loglinear regression analysis was conducted (Figure 3). A negative relationship was noted between the number of formed cyst nematodes and the yield of drought tolerant wheat lines $\left(\mathrm{R}^{2}=0.11\right)$, while heat tolerant wheat lines seem to be more tolerant to the effect of cyst nematode formation as wheat yield values that were positively related to the number of formed cysts $\left(\mathrm{R}^{2}=\right.$ 0.15). Moreover, despite the noted negative effect of the number of formed cysts, the yield of drought tolerant lines was considered to be higher than the heat tolerant lines.

Breeding wheat for resistance against CCNs started in the 1970s (Brown \& Ellis 1976) and has become one of the most effective and desirable methods of control especially against $H$. filipjevi to prevent yield losses. The use of resistant lines is desirable and the only enduring method present to control CCNs due to its low cost, being user-friendly and is acknowledged to not be harmful to the environment (Dababat et al. 2014; Williamson \& Kumar 2006). Different control methods can be used but have limitations and there are very few reports related to wheat-nematode interaction. Goverse \& Smant (2018) pointed out that the complete mechanism of resistance is still an enigma with incomplete knowledge on plant immunity to plant-parasitic nematodes. Specific genes for the resistance against $H$. filipjevi are yet to be identified despite some of the Cre genes have shown certain degrees of success against the nematode such as; Cre8 and CreR which showed some levels of resistance (Imren et al. 2012). Toktay et al. (2012) screened resistant wheat lines containing the Crel gene which showed different resistance responses to $H$. filipjevi. It has been recognized that some of the identified 12 Cre genes that are known as a resistant source in wheat to $H$. 
avenae can show resistance to $H$. filipjevi and have shown success against other CCNs (Blok et al. 2018). To date, 16 different resistance genes to $\mathrm{H}$. avenae, including $12 \mathrm{Cre}$ genes; Cre1, Cre2, Cre3, Cre4, Cre5, Cre6, Cre 7, Cre8, Cre $9 \mathrm{CreR}, \mathrm{CreY}$ and $\mathrm{Cre} 3 \mathrm{~S}$ in wheat and its wild relatives and $\mathrm{Hal}, \mathrm{Ha}$, $\mathrm{Ha}$ 3, $\mathrm{Ha} 4$ genes in barley were reported (Bakker et al. 2006; Zhai et al. 2008; Smiley \& Nicol 2009; Moens et al. 2018; Cui et al. 2020), which also might have resistance to H. filipjevi. Kimber \& Feldman (1987) mentioned that wheat varieties showing resistance or tolerance responses have shown to provide resistance against a wide range of biotic and abiotic stress factors. There are some assumptions that there might be a strong connection between drought and heat tolerance with CCN resistance and is yet to be proven. Dababat et al. (2018) has addressed this matter, as it was mentioned that water stressed crops grown in arid and semi-arid regions that have the ability to secure adequate amounts of water can be severely weakened due to the effect of nematodes on the crops root system. Correspondingly, with our current findings, it has been found that there is a weak positive correlation between wheat lines with heat tolerance and the number of formed cysts (resistance response), as these lines showed more tolerance without negatively affecting their yield. Our findings also indicated that there is a weak negative correlation between drought tolerant wheat lines and number of formed cysts. This is most likely due to the genetic background of these lines.

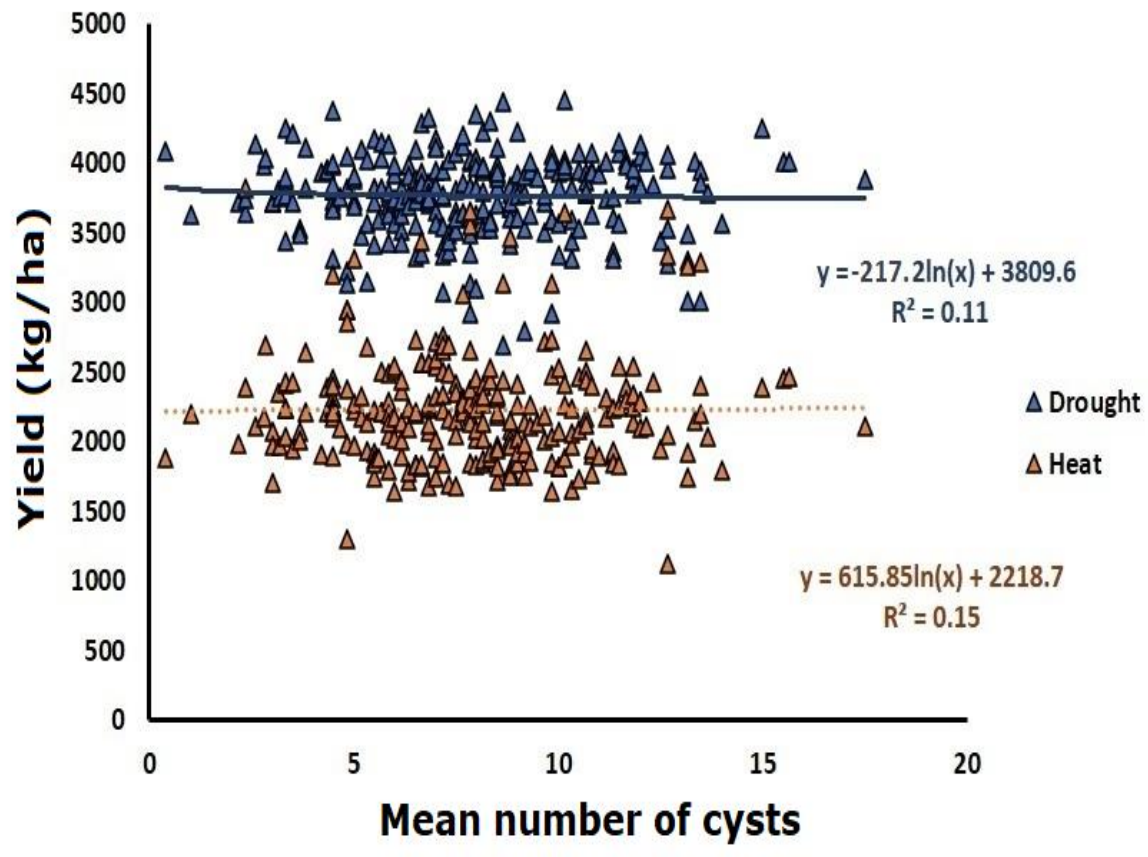

Figure 3- Log-linear regressions of wheat yield and mean number of formed cysts in 2 experiments. All $R^{2}$ values were significant at $P<0.05$. Values are means of 1 years, each with 6 replicates per line. The link represents the predicted logarithmic regression model. Equations were represented on $\mathrm{kg} \mathrm{ha}^{-1}$

The study has managed to find 11 resistant and 36 moderately resistant spring wheat lines, noting that the screened wheat set originates from a diverse genetic background. Different screening studies that have been done on wheat accessions originating from different sources have shown resistance to H. filipjevi (Toktay et al. 2012; Dababat et al. 2014; Pariyar et al. 2016a, b; Yavuzaslanoglu et al. 2016; Dababat 2019)

There is a difficulty to truly compare the result of this study to other similar or related studies despite using the same experimental setup, as there are a lot of variables between the experiments. One of the main reasons regarding the difficulty in comparison is due to different categorizing of the resistant response groups that rely on the average number of formed cysts and females on the root system per plant and comparing them to the check wheat cultivars with known resistance response. Pariyar et al. (2016b) and Yavuzaslanoglu et al. (2016) have used a different arrangement of the average number of formed cysts and females/plant assigned to the resistant groups with reliance on the check wheat cultivars response. Zhang et al. (2012) even used a different method of sorting which relied on the relative resistance index (RRI); RRI = [1 - (the mean number of white females per plant on a tested line/the mean number of white females per Wenmai 19 check plant).

Also, a point of difference is the experiment setup conditions, Dababat (2019) conducted his experiment under field conditions, Hajihasani et al. (2010) experiment was conducted in pots under field conditions, while Zhang et al. (2012) conducted his experiment in greenhouse conditions. Toktay et al. (2012) and (Pariyar et al. 2016a, b) conducted their study in a growth chamber under a controlled condition and with resembling methodology, so this can be a point of similarity to compare the results.

Generally, when screening wheat accessions for their resistant response almost all studies have obtained a low percentage of resistant accessions from the total screening. This study managed to find a total of $4.28 \%$ resistant lines from 257 lines, Dababat 
(2019) tested 35 resistant lines that were obtained from the previous screening of thousands of wheat accessions. Pariyar et al. (2016a) found only $1 \%$ resistant accessions from a total of 161 accessions and in another study by Pariyar et al. (2016b) found only $1 \%$ resistance of wheat accessions from a total of 291 accessions.

In this type of study, it is expected to find a very low percentage of resistance among the screened accessions. When comparing the results of this study in terms of the percentage of resistance accessions found with the other studies, this study is considered to have a noticeably high percentage of success.

A possible reason for our current finding is the possibility that resistant and moderately resistant lines may have a relationship with the drought and heat tolerant QTLs as assumed. The screened set may also contain a source of Cre genes; like Cre 1 as in Toktay et al. (2012) study, Cre8 or CreR in Imren et al. (2012) study, Cre5 as in Dababat et al. (2014) study, or the same QTLs Pariyar et al. (2016a) has identified or due to the presence of new sources of resistance. This matter cannot be confirmed in this study but future analysis is required to obtain a clearer idea of why these specific lines showed a resistant response to $H$. filipjevi.

\section{Conclusions}

It is notable that despite the abundance of screening studies related to nematode resistance, this study is the first to evaluate the resistant response of $H$. filipjevi against wheat genotyped with drought and heat tolerance. It has been managed to add 11 wheat lines with resistance and 36 lines with moderate resistance to $H$. filipjevi as genetic resources for future wheat breeding programs. This might be good for helping advance resistance studies to CCNs in general but specifically to improve resistance against $H$. filipjevi. It is expected that the study can provide supplementary data with previous work by Pariyar et al. (2016a) for future studies concerned with finding resistant genes to $H$. filipjevi.

Although resistant and moderately resistant lines to $H$. filipjevi were found, it should be noted that further assessment of these lines is recommended to fully verify their resistant and moderately resistant status. Also, it is recommended that these lines should be screened for resistance response to other Heterodera species, mainly H. avenae and H. latipons to obtain wheat lines with resistance to more than one Heterodera species.

This study supports Dababat et al. (2018) remarks, statistically, that there is a correlation between drought and heat tolerance with CCN resistance despite being a weak one but establishes a foundation for more detailed studies.

\section{Acknowledgments}

The authors would like to thank the Transitional Zone Agricultural Research Institute (TZARI) in Eskisehir, Turkey for their technical support.

\section{References}

Afzal F, Chaudhari S K, Gul A, Farooq A, Ali H, Nisar S, Sarfraz B, Shehzadi K J \& Mujeeb-Kazi A (2015). Bread wheat (Triticum aestivum L.) under biotic and abiotic stresses: An overview. In Hakeem K R (Eds), Crop Production and Global Environmental Issues, Springer, pp. 293-317. https://doi.org/10.1007/978-3-319-23162-4_13

Akar T, Caliskan M, Nicol, J M, Uranbey S, Sahin E, Yazar S, William M, \& Braun H J (2009). Molecular characterization of Cereal Cyst Nematode diagnostic markers Crel and Cre3 in some winter wheat germplasm and their potential use against Heterodera filipjevi. Field Crops Research 114(2): 320-323. https://doi.org/10.1016/j.fcr.2009.08.001

Asseng S, Ewert F, Martre P, Rötter R P, Lobell D B, Cammarano D, Kimball B A, Ottman M J, Wall G W, White J W \& Reynolds M P (2015). Rising temperatures reduce global wheat production. Nature climate change 5(2): 143

Bakker E, Dees R, Bakker J \& Goverse A (2006). Mechanisms involved in plant resistance to nematodes. In Tuzun, S. \& Bent, E. (Eds), Multigenic and induced systemic resistance in plants, Springer, Boston, MA, pp. 314-334. https://doi.org/10.1007/0-387-23266-4_14

Blok V C, Tylka G L, Smiley R W, de Jong W S, \& Daub M (2018) Resistance Breeding. In Perry R N, Moens M \& Jones J T (Eds), Cyst Nematodes, CABI, Wallingford, UK, pp. 179. https://doi.org/10.1079/9781786390837.0174

Bridge J \& Starr J (2007). Plant nematodes of agricultural importance: a color handbook. Elsevier.

Brown J A M \& Ellis S E (1976). Breeding for resistance to cereal cyst nematode in wheat. Euphytica 25(1): 73-82. https://doi.org/10.1007/bf00041530

Cui L, Qiu D, Sun L, Sun Y, Ren Y, Zhang H, Li J, Zou J, Wu P, Hu J \& Xie J (2020). Resistance to Heterodera filipjevi and H. avenae in Winter Wheat is Conferred by Different QTL. Phytopathology 110(2): 472-482. https://doi.org/10.1094/phyto-04-19-0135-r

Dababat A A, Amjad Ali M, \& Singh R (2021). The need for Integrated Management of the Cereal Cyst Nematodes, Heterodera avenae and $H$. filipjevi in Central Western Asia and North Africa - CWANA. CABI.

Dababat A A (2019). Resistance and tolerance reactions of winter wheat lines to Heterodera filipjevi in Turkey. Journal of nematology pp. 51. https://doi.org/10.21307/jofnem-2019-031

Dababat A A, Erginbas-Orakci G, Toumi F, Braun H J, Morgounov A I \& Sikora R A (2018). IPM to control soil-borne pests on wheat and sustainable food production. Arab Journal of Plant Protection 36(1): 37-44. https://doi.org/10.22268/ajpp-036.1.037044

Dababat A A \& Fourie H (2018). Nematode parasites of cereals In Sikora R A, Coyne D, Hallmann, J \& Timper P (Eds), Plant Parasitic Nematodes in Subtropical and Tropical Agriculture, CABI, pp. 163-221. https://doi.org/10.1079/9781786391247.0163

Dababat A A, Ferney G B H, Erginbas-Orakci G, Dreisigacker S, Imren M, Toktay H, Elekcioglu H I, Mekete T, Nicol J M, Ansari O \& Ogbonnaya F (2016). Association analysis of resistance to cereal cyst nematodes (Heterodera avenae) and root lesion nematodes 
(Pratylenchus neglectus and P. thornei) in CIMMYT advanced spring wheat lines for semi-arid conditions. Breeding Science pp. 15158. https://doi.org/10.1270/jsbbs. 15158

Dababat A A, Imren M, Erginbas-Orakci G, Ashrafi S, Yavuzaslanoglu E, Toktay H, Pariyar S R, Elekcioglu H I, Morgounov A \& Mekete T (2015). The importance and management strategies of cereal cyst nematodes, Heterodera spp., in Turkey. Euphytica 202(2): 173-188. https://doi.org/10.1007/s10681-014-1269-z

Dababat A A, Pariyar S R, Nicol J M, Erginbas-Orakci G, Goll M, Watrin C, Duveiller E, Braun H J, Cabrera J A \& Sikora R A (2014). Influence of thiabendazole seed treatment on the integrated control of Heterodera filipjevi on six wheat genotypes with different levels of genetic resistance under controlled conditions. Nematropica, 44(1): 25-30

De Fraiture C, Giordano M \& Yongsong L (2007). Biofuels: implications for agricultural water use. Colombo, Sri Lanka: International Water Management Institute.

Droogers P \& Aerts J (2005). Adaptation strategies to climate change and climate variability: a comparative study between seven contrasting river basins. Physics and Chemistry of the Earth, Parts A/B/C, 30(6-7): 339-346. https://doi.org/10.1016/j.pce.2005.06.015

FAOSTAT (2020). Crops. Retrieved in March, 10, 2020 from http://www.fao.org/faostat/en/\#data/QC,.

Goverse A \& Smant G (2018). Mechanisms of Resistance to Cyst Nematodes. In Perry, R.N., Moens, M. \& Jones, J.T. (Eds), Cyst Nematodes, CABI, Wallingford, UK, pp.154. https://doi.org/10.1079/9781786390837.0154

Hajihasani A, Maafi Z T, Nicol J \& Rezaee S (2010). Effect of the cereal cyst nematode, Heterodera filipjevi, on wheat in microplot trials. Nematology 12(3): 357-363. https://doi.org/10.1163/138855409x12548945788321

Handoo Z A (1998). Plant-parasitic nematodes. USDA, ARS, Nematology Laboratory.

Handoo Z A (2002). A key and compendium to species of the Heterodera avenae group (Nematoda: Heteroderidae). Journal of Nematology 34(3): 250

Imren M, Waeyenberge L, Viaene N, Toktay H, Dababat A A \& Elekçİoğlu İ H (2012). Molecular characterization of cereal cyst nematodes from the South Anatolian Region in Turkey using ITS-rDNA sequences. Türkiye Entomoloji Dergisi 36(4): 491-499. https://doi.org/10.3906/tar-1404-56

Imren M, Yildiz Ş, Çiftçi V \& Dababat A A (2019). Effect of cereal cyst nematode Heterodera filipjevi on wheat yields in Turkey. Turkish Journal of Agriculture and Forestry pp. 43. https://doi.org/10.3906/tar-1902-17

Karimipour Fard H, Pourjam E, Tanha M Z \& Safaie N (2018). Assessment of yield loss of wheat cultivars caused by Heterodera filipjevi under field conditions. Journal of Phytopathology 166(5): 299-304. https://doi.org/10.1111/jph.12686

Kimber G \& Feldman M (1987). Wild wheat. An introduction. CABI (353).

Lawlor D W \& Cornic G (2002). Photosynthetic carbon assimilation and associated metabolism in relation to water deficits in higher plants. Plant, cell \& environment 25(2): 275-294. https://doi.org/10.1046/j.0016-8025.2001.00814.x

Lichtenthaler H K (1996). Vegetation stress: an introduction to the stress concept in plants. Journal of plant physiology 148(1-2): 4-14.

McCarter J P (2009). Molecular approaches toward resistance to plant-parasitic nematodes. In Berg R H \& Taylor C G (Eds), Cell Biology of Plant Nematode Parasitism, Springer Science \& Business Media, Berlin, Heidelberg, pp. 239-267. https://doi.org/10.1007/978-3-54085215-5 9

Moens M, Perry R N \& Jones J T (2018). Cyst Nematodes Life Cycle and Economic Importance. In Perry R N, Moens M \& Jones J T (Eds), Cyst Nematodes, CABI, Wallingford, UK, pp. 1-26. https://doi.org/10.1079/9781786390837.0001

Nicol J M \& Rivoal R (2008). Global knowledge and its applications for the integrated control and management of nematodes on wheat. In Ciancio A \& Mukerji K G (Eds), Integrated management and biocontrol of vegetable and grain crops nematodes (Vol. 2), Springer, Netherlands. https://doi.org/10.1007/978-1-4020-6063-2_13

Nicol J M, Bolat N, Sahin E, Tulek A, Yildirim A F, Yorgancilar A, Kaplan A \& Braun H J (2006). The cereal cyst nematode is causing economic damage on rainfed wheat production systems of Turkey. Phytopathology Vol. 96, No. 6, pp. S169-S169, 3340

Nicol J M, Turner S J, Coyne D L, Den N L, Hockland S \& Maafi Z T (2011). Current nematode threats to world agriculture. In Jones J, Gheysen G \& Fenoll C (Eds) Genomics and molecular genetics of plant-nematode interactions, (pp. 21-43). Springer, Dordrecht. https://doi.org/10.1007/978-94-007-0434-3_2

Pariyar S R, Dababat A A, Sannemann W, Erginbas-Orakci G, Elashry A, Siddique S, Morgounov A, Leon J \& Grundler F M (2016a). Genomewide association study in wheat identifies resistance to the cereal cyst nematode Heterodera filipjevi. Phytopathology, 106(10): 1128-1138. https://doi.org/10.1094/phyto-02-16-0054-fi

Pariyar S R, Dababat A A, Siddique S, Erginbas-Orakci G, Elashry A, Morgounov A \& Grundler F M (2016b). Identification and characterisation of resistance to the cereal cyst nematode Heterodera filipjevi in winter wheat. Nematology 18(4): $377-402$. https://doi.org/10.1163/15685411-00002964

Parry M L, Rosenzweig C, Iglesias A, Livermore M \& Fischer G (2004). Effects of climate change on global food production under SRES emissions and socio-economic scenarios. Global environmental change 14(1), pp.53-67. https://doi.org/10.1016/j.gloenvcha.2003.10.008

Rivoal R \& Cook R (1993). Plant parasitic nematodes in temperate agriculture. In Evans K \& Trudgill D L (Eds), No. 632.6513 E9.Seid A, İmren M, Amjad A M, Toumi F, Paulitz T, \& Dababat A A (2021). Genetic Resistance of Wheat Towards Plant-Parasitic Nematodes: Current Status and Future Prospects. Biotech Studies, 30(1), 43-62. https://doi.org/10.38042/biotechstudies.944678

Seid A, İmren M, Amjad AM, Toumi F, Paulitz T, \& Dababat AA (2021).Buğdayın Bitki-Parazit Nematodlarına Karşı Genetik Direnci: Mevcut Durum ve Gelecek Beklentileri. Biyoteknoloji Çalışmalar, 30(1), 43-62. https://doi.org/10.38042/biotechstudies.944678)

Siddiqi M R (2000). Tylenchida: parasites of plants and insects. CABI.

Smiley R W \& Nicol J M (2009). Nematodes which challenge global wheat production. Wheat science and trade pp. 171-187. https://doi.org/10.1002/9780813818832.ch8

Subbotin S A \& Baldwin J (2010). Systematics of Cyst Nematodes (Nematoda: Heteroderinae), Part B. In Systematics of Cyst Nematodes (Nematoda: Heteroderinae), Part B. Brill. https://doi.org/10.1163/ej.9789004164345.i-512

Toktay H, Yavuzaslanoğlu E, İmren M, Nicol J, Elekcioğlu İ H \& Dababat A A (2012). Screening for resistance to Heterodera filipjevi (Madzhidov) Stelter (Tylenchida: Heteroderidae) and Pratylenchus thornei (Sher \& Allen) (Tylenchida: Pratylenchidae) sister lines of spring wheat. Türkiye Entomoloji Dergisi. https://doi.org/10.15832/tbd.76467

Trethowan R M \& Mujeeb-Kazi A (2008). Novel germplasm resources for improving environmental stress tolerance of hexaploid wheat. Crop Science 48(4): 1255-1265. https://doi.org/10.2135/cropsci2007.08.0477

Webster J M (1987). Introduction. In Brown R H, Kerry B R (Eds) Principles and Practice of Nematode Control in Crops. Academic Press: Melbourne, pp. 1-12 
Vaish S (2017). Management of Wheat and Barley Diseases, In Singh D P (Eds). Apple Academic Press, Canada.

Williamson V M \& Kumar A (2006). Nematode resistance in plants: the battle underground. Trends in Genetics 22(7): 396-403. https://doi.org/10.1016/j.tig.2006.05.003

Yavuzaslanoglu E, Elekcioglu I H, Nicol J M \& Sheedy J G (2016). Resistance of Iranian landrace wheat to the cereal cyst nematode, Heterodera filipjevi. Australasian Plant Pathology 45(4): 411-414. https://doi.org/10.1007/s13313-016-0427-2

Zhao C, Liu B, Piao S, Wang X, Lobell D B, Huang Y, Huang M, Yao Y, Bassu S, Ciais P \& Durand J L (2017). Temperature increase reduces global yields of major crops in four independent estimates. In: Proceedings of the National Academy of Sciences, 114(35): 9326-9331. https://doi.org/10.1073/pnas.1701762114

Zhai X G, Zhao T, Liu Y H, Long H, Deng G B, Pan Z F \& Yu M Q (2008). Characterization and expression profiling of a novel cereal cyst nematode resistance gene analog in wheat. Molecular biology 42(6): 960-965. https://doi.org/10.1134/s0026893308060186

Zhang J, Yuan H, Zhang R, Xing X, Niu J, Li H \& Chen P (2012). Analysis of resistance to Heterodera filipjevi in Triticum aestivumDasypyrum villosum germplasm. Acta Agronomica Sinica 38(11): 1969-1976. https://doi.org/10.3724/sp.j.1006.2012.01969

Supplementary Table1: List of Drought and Heat Tolerant Spring Wheat lines and their Resistant Response to the CCN Heterodera filipjevi. (SD) Standard Deviation, (SE) Standard Error, (CID) Cross ID

\begin{tabular}{|c|c|c|c|c|c|c|c|}
\hline $\begin{array}{c}\text { ENTRY } \\
\text { NUMBER }\end{array}$ & $C I D$ & PEDIGREE (CROSS) & $\begin{array}{l}\text { SELECTION } \\
\text { HISTORY }\end{array}$ & $\begin{array}{l}\text { (MEAN) } \\
\text { CYSTS } \\
\text { AND } \\
\text { FEMALES }\end{array}$ & $S D$ & $S E$ & $\begin{array}{l}\text { RESISTANT } \\
\text { RESPONSE }\end{array}$ \\
\hline 265 & 620759 & $\begin{array}{l}\text { PERSIA-88/3/PBW343*2/KUKUNA*2//FRTL/PIFED/4/ } \\
\text { QUAIU \#1 }\end{array}$ & $\begin{array}{l}\text { SDSS13Y00190T-0B- } \\
\text { 0Y-0M-0Y-0B-71Y }\end{array}$ & 0.40 & 0.55 & 3.11 & $\mathrm{R}$ \\
\hline 210 & 620687 & CROC_1/AE.SQUARROSA (436)//KACHU/3/BAJ \#1 & $\begin{array}{l}\text { SDSS13Y00118T-0B- } \\
\text { 0Y-0M-0Y-0B-113Y }\end{array}$ & 1.00 & 0.89 & 0.37 & $\mathrm{R}$ \\
\hline 70 & 620629 & H-1546/NELOKI/3/ATTILA*2/PBW65//MURGA & $\begin{array}{l}\text { SDSS13Y00060T-0B- } \\
\text { 0Y-0M-0Y-0B-31Y }\end{array}$ & 2.17 & 1.33 & 1.14 & $\mathrm{R}$ \\
\hline 203 & 620683 & H-1601/NAVJ07//KACHU & $\begin{array}{l}\text { SDSS13Y00114T-0B- } \\
\text { 0Y-0M-0Y-0B-106Y }\end{array}$ & 2.33 & 1.21 & 0.84 & $\mathrm{R}$ \\
\hline 264 & 620749 & IG 122743/NAVJ07//KACHU & $\begin{array}{l}\text { SDSS13Y00180T-0B- } \\
\text { 0Y-0M-0Y-0B-33Y }\end{array}$ & 2.33 & 0.82 & 0.33 & $\mathrm{R}$ \\
\hline 54 & 620613 & $\begin{array}{l}\text { IG 1505/4/PRL/2*PASTOR//PBW343*2/KUKUNA/3/ } \\
\text { ROLF07/5/NELOKI }\end{array}$ & $\begin{array}{l}\text { SDSS13Y00044T-0B- } \\
\text { 0Y-0M-0Y-0B-31Y }\end{array}$ & 2.60 & 2.41 & 2.56 & $\mathrm{R}$ \\
\hline 49 & 620609 & $\begin{array}{l}\text { DOY1/AE.SQUARROSA (447)/4/PRL/2*PASTOR// } \\
\text { PBW343*2/KUKUNA/3/ROLF07/5/NELOKI }\end{array}$ & $\begin{array}{l}\text { SDSS13Y00040T-0B- } \\
\text { 0Y-0M-0Y-0B-43Y }\end{array}$ & 2.80 & 2.28 & 2.83 & $\mathrm{R}$ \\
\hline 263 & 620748 & IG 122741/NAVJ07//KACHU & $\begin{array}{l}\text { SDSS13Y00179T-0B- } \\
\text { 0Y-0M-0Y-0B-28Y }\end{array}$ & 2.83 & 1.72 & 1.14 & $\mathrm{R}$ \\
\hline 42 & 620600 & $\begin{array}{l}\text { H-1357/8/CNDO/R143//ENTE/MEXI_2/3/AEGILOPS } \\
\text { SQUARROSA (TAUS)/4/WEAVER/5/PICUS/6/TROST } \\
\text { /7/TACUPETO F2001/9/KAUZ//ALTAR 84/AOS/3/ } \\
\text { PASTOR/4/MILAN/CUPE//SW89.3064/5/KIRITATI }\end{array}$ & $\begin{array}{l}\text { SDSS13Y00031T-0B- } \\
\text { 0Y-0M-0Y-0B-41Y }\end{array}$ & 3.00 & 1.79 & 1.32 & $\mathrm{R}$ \\
\hline 251 & 620729 & IG 122139/NAVJ07//KACHU & $\begin{array}{l}\text { SDSS13Y00160T-0B- } \\
\text { 0Y-0M-0Y-0B-38Y }\end{array}$ & 3.00 & 1.90 & 1.18 & $\mathrm{R}$ \\
\hline 254 & 620736 & $\begin{array}{l}\text { IG 122627/6/KAUZ//ALTAR 84/AOS/3/PASTOR/4/ } \\
\text { MILAN/CUPE//SW89.3064/5/KIRITATI/7/SW89.5277/ } \\
\text { BORL95//SKAUZ/3/PRL/2*PASTOR/4/HEILO }\end{array}$ & $\begin{array}{l}\text { SDSS13Y00167T-0B- } \\
\text { 0Y-0M-0Y-0B-38Y }\end{array}$ & 3.00 & 1.41 & 1.51 & $\mathrm{R}$ \\
\hline 128 & 620641 & $\begin{array}{l}\text { 68.111/RGB-U//WARD RESEL/3/STIL/4/AE. } \\
\text { SQUARROSA (630)/5/BORL14/6/COPIO }\end{array}$ & $\begin{array}{l}\text { SDSS13Y00072T-0B- } \\
\text { 0Y-0M-0Y-0B-58Y }\end{array}$ & 3.17 & 1.60 & 0.65 & MR \\
\hline $\begin{array}{l}\text { KATEA } \\
\text { (MR) }\end{array}$ & & KHEBROS/BEZOSTAYA-1 & & 3.17 & 2.71 & 1.11 & MR \\
\hline 81 & 620631 & H-1699/NELOKI/3/ATTILA*2/PBW65//MURGA & $\begin{array}{l}\text { SDSS13Y00062T-0B- } \\
\text { 0Y-0M-0Y-0B-23Y }\end{array}$ & 3.20 & 2.28 & 2.76 & MR \\
\hline 69 & 620629 & H-1546/NELOKI/3/ATTILA*2/PBW65//MURGA & $\begin{array}{l}\text { SDSS13Y00060T-0B- } \\
\text { 0Y-0M-0Y-0B-30Y }\end{array}$ & 3.33 & 1.97 & 0.80 & MR \\
\hline 157 & 620652 & PERSIA-7/COPIO/3/KACHU \#1/KIRITATI//KACHU & $\begin{array}{l}\text { SDSS13Y00083T-0B- } \\
\text { 0Y-0M-0Y-0B-65Y }\end{array}$ & 3.33 & 2.07 & 2.03 & MR \\
\hline 225 & 620690 & GAN/AE.SQUARROSA (206)//KACHU/3/BAJ \#1 & $\begin{array}{l}\text { SDSS13Y00121T-0B- } \\
\text { 0Y-0M-0Y-0B-83Y }\end{array}$ & 3.33 & 1.51 & 1.41 & MR \\
\hline 250 & 620729 & IG 122139/NAVJ07//KACHU & $\begin{array}{l}\text { SDSS13Y00160T-0B- } \\
\text { 0Y-0M-0Y-0B-34Y }\end{array}$ & 3.33 & 2.07 & 1.58 & MR \\
\hline 257 & 620746 & $\begin{array}{l}\text { IG 122738/3/PBW343*2/KUKUNA*2//FRTL/ } \\
\text { PIFED/4/QUAIU \#1 }\end{array}$ & $\begin{array}{l}\text { SDSS13Y00177T-0B- } \\
\text { 0Y-0M-0Y-0B-54Y }\end{array}$ & 3.33 & 2.16 & 0.88 & MR \\
\hline 57 & 620615 & $\begin{array}{l}\text { IG 122145/4/PRL/2*PASTOR//PBW343*2/KUKUNA/3/ } \\
\text { ROLF07/5/NELOKI }\end{array}$ & $\begin{array}{l}\text { SDSS13Y00046T-0B- } \\
\text { 0Y-0M-0Y-0B-38Y }\end{array}$ & 3.50 & 2.35 & 1.41 & MR \\
\hline 256 & 620736 & $\begin{array}{l}\text { IG 122627/6/KAUZ//ALTAR 84/AOS/3/PASTOR } \\
\text { /4/MILAN/CUPE//SW89.3064/5/KIRITATI/7/SW89.527 } \\
\text { 7/BORL95//SKAUZ/3/PRL/2*PASTOR/4/HEILO }\end{array}$ & $\begin{array}{l}\text { SDSS13Y00167T-0B- } \\
\text { 0Y-0M-0Y-0B-43Y }\end{array}$ & 3.50 & 1.64 & 1.28 & MR \\
\hline 208 & 620687 & CROC_1/AE.SQUARROSA (436)//KACHU/3/BAJ \#1 & $\begin{array}{l}\text { SDSS13Y00118T-0B- } \\
\text { 0Y-0M-0Y-0B-101Y }\end{array}$ & 3.67 & 4.97 & 2.03 & MR \\
\hline 261 & 620748 & IG 122741/NAVJ07//KACHU & $\begin{array}{l}\text { SDSS13Y00179T-0B- } \\
\text { 0Y-0M-0Y-0B-22Y }\end{array}$ & 3.67 & 2.50 & 1.23 & MR \\
\hline $\begin{array}{l}\text { SONMEZ } \\
\text { (MR) }\end{array}$ & & $\begin{array}{l}\text { BEZOSTAYA-1//BEZOSTAYA-1/TEVERE/3/ } \\
\text { KREMENA/LOVRIN-29/4/KATYA-1[3669] }\end{array}$ & & 3.80 & 2.17 & 0.97 & MR \\
\hline 87 & 620633 & IG 131672/3/ATTILA*2/PBW65//MURGA/4/BORL14 & $\begin{array}{l}\text { SDSS13Y00064T-0B- } \\
\text { 0Y-0M-0Y-0B-60Y }\end{array}$ & 3.83 & 2.23 & 1.68 & MR \\
\hline 227 & 620693 & $\begin{array}{l}\text { D67.2/PARANA 66.270//AE.SQUARROSA (506)/3/ } \\
\text { KACHU/4/BAJ \#1 }\end{array}$ & $\begin{array}{l}\text { SDSS13Y00124T-0B- } \\
\text { 0Y-0M-0Y-0B-49Y }\end{array}$ & 3.83 & 1.47 & 0.60 & MR \\
\hline
\end{tabular}


Supplementary Table2(Continue): List of Drought and Heat Tolerant Spring Wheat lines and their Resistant Response to the CCN Heterodera filipjevi. (SD) Standard Deviation, (SE) Standard Error, (CID) Cross ID

\begin{tabular}{|c|c|c|c|c|c|c|c|}
\hline $\begin{array}{c}E N T R Y \\
N U M B E R\end{array}$ & $C I D$ & PEDIGREE (CROSS) & $\begin{array}{l}\text { SELECTION } \\
\text { HISTORY }\end{array}$ & $\begin{array}{c}\text { (MEAN) } \\
\text { CYSTS } \\
\text { AND } \\
\text { FEMALES }\end{array}$ & $S D$ & $S E$ & $\begin{array}{l}\text { RESISTANT } \\
\text { RESPONSE }\end{array}$ \\
\hline 174 & 620655 & $\begin{array}{l}\text { CETA/AE.SQUARROSA }(872) / 3 / \mathrm{KACHU} \# 1 / \\
\text { KIRITATI/KACHU/4/PBW343*2/KUKUNA } 2 / / \text { FRT } \\
\text { L/PIFED }\end{array}$ & $\begin{array}{l}\text { SDSS13Y00086T-0B- } \\
\text { 0Y-0M-0Y-0B-58Y }\end{array}$ & 4.20 & 2.49 & 3.58 & MR \\
\hline 143 & 620646 & IWA8611400/BORL14//COPIO & $\begin{array}{l}\text { SDSS13Y00077T-0B- } \\
\text { 0Y-0M-0Y-0B-112Y }\end{array}$ & 4.33 & 2.66 & 1.09 & MR \\
\hline 219 & 620689 & CROC_1/AE.SQUARROSA (176)//KACHU/3/BAJ \#1 & $\begin{array}{l}\text { SDSS13Y00120T-0B- } \\
\text { 0Y-0M-0Y-0B-87Y }\end{array}$ & 4.33 & 2.73 & 1.63 & MR \\
\hline 127 & 620641 & $\begin{array}{l}\text { 68.111/RGB-U//WARD RESEL/3/STIL/4/AE. } \\
\text { SQUARROSA (630)/5/BORL14/6/COPIO }\end{array}$ & $\begin{array}{l}\text { SDSS13Y00072T-0B- } \\
\text { 0Y-0M-0Y-0B-57Y }\end{array}$ & 4.50 & 2.95 & 1.54 & MR \\
\hline 144 & 620646 & IWA8611400/BORL14//COPIO & $\begin{array}{l}\text { SDSS13Y00077T-0B- } \\
\text { 0Y-0M-0Y-0B-113Y }\end{array}$ & 4.50 & 3.02 & 1.89 & MR \\
\hline 145 & 620647 & $\begin{array}{l}\text { T.DICOCCON PI94624/AE.SQUARROSA (454)// } \\
\text { COPIO/3/KACHU \#1/KIRITATI/KACHU }\end{array}$ & $\begin{array}{l}\text { SDSS13Y00078T-0B- } \\
\text { 0Y-0M-0Y-0B-71Y }\end{array}$ & 4.50 & 2.74 & 1.69 & MR \\
\hline 168 & 620653 & PERSIA-21/COPIO/3/KACHU \#1/KIRITATI/KACHU & $\begin{array}{l}\text { SDSS13Y00084T-0B- } \\
\text { 0Y-0M-0Y-0B-75Y }\end{array}$ & 4.50 & 2.88 & 1.61 & MR \\
\hline 169 & 620653 & PERSIA-21/COPIO/3/KACHU \#1/KIRITATI//KACHU & $\begin{array}{l}\text { SDSS13Y00084T-0B- } \\
\text { 0Y-0M-0Y-0B-77Y }\end{array}$ & 4.50 & 2.81 & 1.96 & MR \\
\hline 189 & 620665 & $\begin{array}{l}\text { GARZA/BOY//AE.SQUARROSA (281)/3/ } \\
\text { PBW343*2/KUKUNA*2//FRTL/PIFED/4/QUAIU \#1 }\end{array}$ & $\begin{array}{l}\text { SDSS13Y00096T-0B- } \\
\text { 0Y-0M-0Y-0B-62Y }\end{array}$ & 4.50 & 2.81 & 1.34 & MR \\
\hline 199 & 620680 & IG 41243/NAVJ07//KACHU & $\begin{array}{l}\text { SDSS13Y00111T-0B- } \\
\text { 0Y-0M-0Y-0B-58Y }\end{array}$ & 4.50 & 2.88 & 1.18 & MR \\
\hline 239 & 620699 & $\begin{array}{l}\text { IG 41506/3/PBW343*2/KUKUNA*2//FRTL/ } \\
\text { PIFED/4/QUAIU \#1 }\end{array}$ & $\begin{array}{l}\text { SDSS13Y00130T-0B- } \\
\text { 0Y-0M-0Y-0B-71Y }\end{array}$ & 4.50 & 3.15 & 1.28 & MR \\
\hline 247 & 620718 & IG 43238/NAVJ07//KACHU & $\begin{array}{l}\text { SDSS13Y00149T-0B- } \\
\text { 0Y-0M-0Y-0B-5Y }\end{array}$ & 4.50 & 2.88 & 1.48 & MR \\
\hline 273 & 620785 & IWA8614378/NAVJ07//KACHU & $\begin{array}{l}\text { SDSS13Y00216T-0B- } \\
\text { 0Y-0M-0Y-0B-119Y }\end{array}$ & 4.50 & 2.35 & 0.96 & MR \\
\hline $\begin{array}{l}\text { SONMEZ } \\
\text { (MR) }\end{array}$ & & $\begin{array}{l}\text { BEZOSTAYA-1//BEZOSTAYA-1/TEVERE/3/ } \\
\text { KREMENA/LOVRIN-29/4/KATYA-1[3669] }\end{array}$ & & 4.50 & 2.07 & 0.85 & MR \\
\hline 160 & 620652 & PERSIA-7/COPIO/3/KACHU \#1/KIRITATI//KACHU & $\begin{array}{l}\text { SDSS13Y00083T-0B- } \\
\text { 0Y-0M-0Y-0B-71Y }\end{array}$ & 4.67 & 2.34 & 0.95 & MR \\
\hline 170 & 620653 & PERSIA-21/COPIO/3/KACHU \#1/KIRITATI//KACHU & $\begin{array}{l}\text { SDSS13Y00084T-0B- } \\
\text { 0Y-0M-0Y-0B-81Y }\end{array}$ & 4.83 & 3.37 & 1.92 & MR \\
\hline 215 & 620687 & CROC_1/AE.SQUARROSA (436)//KACHU/3/BAJ \#1 & $\begin{array}{l}\text { SDSS13Y00118T-0B- } \\
\text { 0Y-0M-0Y-0B-137Y }\end{array}$ & 4.83 & 4.45 & 1.82 & MR \\
\hline 229 & 620694 & INDIA-59/KACHU//BAJ \#1 & $\begin{array}{l}\text { SDSS13Y00125T-0B- } \\
\text { 0Y-0M-0Y-0B-79Y }\end{array}$ & 4.83 & 2.56 & 1.05 & MR \\
\hline 246 & 620710 & IG 41735/NAVJ07//KACHU & $\begin{array}{l}\text { SDSS13Y00141T-0B- } \\
\text { 0Y-0M-0Y-0B-78Y }\end{array}$ & 4.83 & 4.79 & 1.96 & MR \\
\hline 278 & 620786 & $\begin{array}{l}\text { IWA8612701/6/KAUZ//ALTAR 84/AOS/3/PASTOR } \\
\text { /4/MILAN/CUPE//SW89.3064/5/KIRITATI/7/SW89.527 } \\
\text { 7/BORL95//SKAUZ/3/PRL/2*PASTOR/4/HEILO }\end{array}$ & $\begin{array}{l}\text { SDSS13Y00217T-0B- } \\
\text { 0Y-0M-0Y-0B-75Y }\end{array}$ & 4.83 & 2.32 & 0.95 & MR \\
\hline 165 & 620652 & PERSIA-7/COPIO/3/KACHU \#1/KIRITATI/KACHU & $\begin{array}{l}\text { SDSS13Y00083T-0B- } \\
\text { 0Y-0M-0Y-0B-79Y }\end{array}$ & 5.00 & 2.76 & 1.53 & MR \\
\hline 166 & 620652 & PERSIA-7/COPIO/3/KACHU \#1/KIRITATI//KACHU & $\begin{array}{l}\text { SDSS13Y00083T-0B- } \\
\text { 0Y-0M-0Y-0B-81Y }\end{array}$ & 5.00 & 2.76 & 1.13 & MR \\
\hline 221 & 620689 & CROC_1/AE.SQUARROSA (176)//KACHU/3/BAJ \#1 & $\begin{array}{l}\text { SDSS13Y00120T-0B- } \\
\text { 0Y-0M-0Y-0B-100Y }\end{array}$ & 5.00 & 3.03 & 1.81 & MR \\
\hline 241 & 620699 & $\begin{array}{l}\text { IG 41506/3/PBW343*2/KUKUNA*2// } \\
\text { FRTL/PIFED/4/QUAIU \#1 }\end{array}$ & $\begin{array}{l}\text { SDSS13Y00130T-0B- } \\
\text { 0Y-0M-0Y-0B-75Y }\end{array}$ & 5.00 & 5.14 & 2.10 & MR \\
\hline $\begin{array}{c}\text { KATEA } \\
(\text { MR) }\end{array}$ & & KHEBROS/BEZOSTAYA-1 & & 5.00 & 2.58 & 1.29 & MR \\
\hline 20 & 620583 & H-1624/BAJ \#1//SUP152 & $\begin{array}{l}\text { SDSS13Y00014T-0B- } \\
\text { 0Y-0M-0Y-0B-32Y }\end{array}$ & 5.17 & 2.64 & 1.97 & MS \\
\hline 29 & 620588 & $\begin{array}{l}\text { H-1311/3/FRET2*2/SHAMA//KACHU/4/ } \\
\text { HUW234+LR34/PRINIA*2//KIRITATI }\end{array}$ & $\begin{array}{l}\text { SDSS13Y00019T-0B- } \\
\text { 0Y-0M-0Y-0B-4Y }\end{array}$ & 5.17 & 2.32 & 0.95 & MS \\
\hline 30 & 620588 & $\begin{array}{l}\text { H-1311/3/FRET2*2/SHAMA//KACHU/4/ } \\
\text { HUW234+LR34/PRINIA*2//KIRITATI }\end{array}$ & $\begin{array}{l}\text { SDSS13Y00019T-0B- } \\
\text { 0Y-0M-0Y-0B-7Y }\end{array}$ & 5.33 & 3.14 & 1.61 & MS \\
\hline 114 & 620640 & $\begin{array}{l}\text { 68.111/RGB-U//WARD } \\
\text { RESEL/3/STIL/4/AE.SQUARROSA } \\
\text { (628)/5/BORL14/6/COPIO }\end{array}$ & $\begin{array}{l}\text { SDSS13Y00071T-0B- } \\
\text { 0Y-0M-0Y-0B-46Y }\end{array}$ & 5.33 & 3.61 & 1.48 & MS \\
\hline 137 & 620645 & IWA8612416/BORL14//COPIO & $\begin{array}{l}\text { SDSS13Y00076T-0B- } \\
\text { 0Y-0M-0Y-0B-46Y }\end{array}$ & 5.33 & 3.01 & 1.91 & MS \\
\hline 244 & 620710 & IG 41735/NAVJ07//KACHU & $\begin{array}{l}\text { SDSS13Y00141T-0B- } \\
\text { 0Y-0M-0Y-0B-67Y }\end{array}$ & 5.33 & 2.80 & 1.15 & MS \\
\hline 148 & 620648 & $\begin{array}{l}\text { T.DICOCCON PI94625/AE.SQUARROSA (372)// } \\
\text { COPIO/3/KACHU \#1/KIRITATI//KACHU }\end{array}$ & $\begin{array}{l}\text { SDSS13Y00079T-0B- } \\
\text { 0Y-0M-0Y-0B-102Y }\end{array}$ & 5.50 & 3.15 & 1.61 & MS \\
\hline 159 & 620652 & PERSIA-7/COPIO/3/KACHU \#1/KIRITATI/KACHU & $\begin{array}{l}\text { SDSS13Y00083T-0B- } \\
\text { 0Y-0M-0Y-0B-69Y }\end{array}$ & 5.50 & 3.78 & 1.54 & MS \\
\hline 184 & 620662 & $\begin{array}{l}\text { YAV79//DACK/RABI/3/SNIPE/4/AE.SQUARROSA } \\
\text { (477)/5/KACHU \#1/KIRITATI//KACHU/6/PBW343*2/ } \\
\text { KUKUNA*2//FRTL/PIFED }\end{array}$ & $\begin{array}{l}\text { SDSS13Y00093T-0B- } \\
\text { 0Y-0M-0Y-0B-87Y }\end{array}$ & 5.50 & 6.47 & 2.64 & MS \\
\hline
\end{tabular}


Supplementary Table3(Continue): List of Drought and Heat Tolerant Spring Wheat lines and their Resistant Response to the CCN Heterodera filipjevi. (SD) Standard Deviation, (SE) Standard Error, (CID) Cross ID

\begin{tabular}{|c|c|c|c|c|c|c|c|}
\hline $\begin{array}{c}\text { ENTRY } \\
\text { NUMBER }\end{array}$ & $C I D$ & PEDIGREE (CROSS) & $\begin{array}{l}\text { SELECTION } \\
\text { HISTORY }\end{array}$ & $\begin{array}{c}\text { (MEAN) } \\
\text { CYSTS } \\
\text { AND } \\
\text { FEMALES }\end{array}$ & $S D$ & $S E$ & $\begin{array}{l}\text { RESISTANT } \\
\text { RESPONSE }\end{array}$ \\
\hline 262 & 620748 & IG 122741/NAVJ07//KACHU & $\begin{array}{l}\text { SDSS13Y00179T-0B- } \\
\text { 0Y-0M-0Y-0B-27Y }\end{array}$ & 5.50 & 2.59 & 1.06 & MS \\
\hline 271 & 620783 & IWA8612134/NAVJ07//KACHU & $\begin{array}{l}\text { SDSS13Y00214T-0B- } \\
\text { 0Y-0M-0Y-0B-88Y }\end{array}$ & 5.50 & 3.78 & 1.89 & MS \\
\hline 110 & 620638 & $\begin{array}{l}\text { LOCAL RED/AE.SQUARROSA (223)// } \\
\text { BORL14/3/COPIO }\end{array}$ & $\begin{array}{l}\text { SDSS13Y00069T-0B- } \\
\text { 0Y-0M-0Y-0B-62Y }\end{array}$ & 5.60 & 3.85 & 3.83 & MS \\
\hline 47 & 620603 & $\begin{array}{l}\text { IG 42147/6/KAUZ//ALTAR 84/AOS/3/PASTOR/4/ } \\
\text { MILAN/CUPE//SW89.3064/5/KIRITATI/7/SW89.5277/ } \\
\text { BORL95//SKAUZ/3/PRL/2*PASTOR/4/HEILO }\end{array}$ & $\begin{array}{l}\text { SDSS13Y00034T-0B- } \\
\text { 0Y-0M-0Y-0B-48Y }\end{array}$ & 5.67 & 3.20 & 1.31 & MS \\
\hline 181 & 620661 & $\begin{array}{l}\text { YAV79//DACK/RABI/3/SNIPE/4/AE.SQUARROSA } \\
\text { (460)/5/KACHU \#1/KIRITATI//KACHU/6/ } \\
\text { PBW343*2/KUKUNA*2//FRTL/PIFED }\end{array}$ & $\begin{array}{l}\text { SDSS13Y00092T-0B- } \\
\text { 0Y-0M-0Y-0B-99Y }\end{array}$ & 5.67 & 3.56 & 1.45 & MS \\
\hline 234 & 620695 & INDIA-107/KACHU//BAJ \#1 & $\begin{array}{l}\text { SDSS13Y00126T-0B- } \\
\text { 0Y-0M-0Y-0B-108Y }\end{array}$ & 5.67 & 3.83 & 1.56 & MS \\
\hline 88 & 620633 & IG 131672/3/ATTILA*2/PBW65//MURGA/4/BORL14 & $\begin{array}{l}\text { SDSS13Y00064T-0B- } \\
\text { 0Y-0M-0Y-0B-70Y }\end{array}$ & 5.83 & 3.76 & 1.92 & MS \\
\hline 99 & 620635 & INDIA-50/3/ATTILA*2/PBW65//MURGA/4/BORL14 & $\begin{array}{l}\text { SDSS13Y00066T-0B- } \\
\text { 0Y-0M-0Y-0B-62Y }\end{array}$ & 5.83 & 3.71 & 1.51 & MS \\
\hline 216 & 620687 & CROC_1/AE.SQUARROSA (436)//KACHU/3/BAJ \#1 & $\begin{array}{l}\text { SDSS13Y00118T-0B- } \\
\text { 0Y-0M-0Y-0B-139Y }\end{array}$ & 5.83 & 4.17 & 1.70 & MS \\
\hline 248 & 620726 & $\begin{array}{l}\text { IG 107128/4/PRL/2*PASTOR//PBW343*2/ } \\
\text { KUKUNA/3/ROLF07/5/NELOKI }\end{array}$ & $\begin{array}{l}\text { SDSS13Y00157T-0B- } \\
\text { 0Y-0M-0Y-0B-47Y }\end{array}$ & 5.83 & 3.60 & 1.68 & MS \\
\hline 270 & 620777 & IWA 8602098/NAVJ07//KACHU & $\begin{array}{l}\text { SDSS13Y00208T-0B- } \\
\text { 0Y-0M-0Y-0B-46Y }\end{array}$ & 5.83 & 5.95 & 2.43 & MS \\
\hline 272 & 620785 & IWA8614378/NAVJ07//KACHU & $\begin{array}{l}\text { SDSS13Y00216T-0B- } \\
\text { 0Y-0M-0Y-0B-116Y }\end{array}$ & 5.83 & 4.02 & 2.32 & MS \\
\hline 33 & 620594 & $\begin{array}{l}\text { INDIA-223/7/SHA7/VEE\#5/5/VEE\#8//JUP/BJY/3/ } \\
\text { F3.71/TRM/4/2*WEAVER/6/SKAUZ/PARUS//PARUS/ } \\
\text { 8/CNDO/R143//ENTE/MEXI_2/3/AEGILOPS } \\
\text { SQUARROSA (TAUS)/4/WEAVER/5/PICUS/6/ } \\
\text { TROST/7/TACUPETO F2001 }\end{array}$ & $\begin{array}{l}\text { SDSS13Y00025T-0B- } \\
\text { 0Y-0M-0Y-0B-24Y }\end{array}$ & 6.00 & 6.51 & 2.66 & MS \\
\hline 41 & 620600 & $\begin{array}{l}\text { H-1357/8/CNDO/R143//ENTE/MEXI_2/3/AEGILOPS } \\
\text { SQUARROSA (TAUS)/4/WEAVER/5/PICUS/6/ } \\
\text { TROST/7/TACUPETO F2001/9/KAUZ//ALTAR 84 } \\
\text { /AOS/3/PASTOR/4/MILAN/CUPE//SW89.3064/5/ } \\
\text { KIRITATI }\end{array}$ & $\begin{array}{l}\text { SDSS13Y00031T-0B- } \\
\text { 0Y-0M-0Y-0B-40Y }\end{array}$ & 6.00 & 6.39 & 2.61 & MS \\
\hline 67 & 620629 & H-1546/NELOKI/3/ATTILA*2/PBW65//MURGA & $\begin{array}{l}\text { SDSS13Y00060T-0B- } \\
\text { 0Y-0M-0Y-0B-23Y }\end{array}$ & 6.00 & 8.27 & 3.38 & MS \\
\hline 202 & 620683 & H-1601/NAVJ07//KACHU & $\begin{array}{l}\text { SDSS13Y00114T-0B- } \\
\text { 0Y-0M-0Y-0B-104Y }\end{array}$ & 6.00 & 4.15 & 2.35 & MS \\
\hline 222 & 620690 & GAN/AE.SQUARROSA (206)//KACHU/3/BAJ \#1 & $\begin{array}{l}\text { SDSS13Y00121T-0B- } \\
\text { 0Y-0M-0Y-0B-54Y }\end{array}$ & 6.00 & 3.95 & 1.61 & MS \\
\hline 204 & 620684 & MEX94.30.10/NAVJ07//KACHU & $\begin{array}{l}\text { SDSS13Y00115T-0B- } \\
\text { 0Y-0M-0Y-0B-98Y }\end{array}$ & 6.17 & 3.37 & 1.38 & MS \\
\hline 206 & 620685 & ARLIN/AE.SQUARROSA (283)//KACHU/3/BAJ \#1 & $\begin{array}{l}\text { SDSS13Y00116T-0B- } \\
\text { 0Y-0M-0Y-0B-68Y }\end{array}$ & 6.17 & 4.26 & 1.74 & MS \\
\hline 218 & 620688 & $\begin{array}{l}\text { AE.SQUARROSA (1029)/DVERD_2//KACHU/3/BAJ } \\
\# 1\end{array}$ & $\begin{array}{l}\text { SDSS13Y00119T-0B- } \\
\text { 0Y-0M-0Y-0B-69Y }\end{array}$ & 6.17 & 3.97 & 2.06 & MS \\
\hline 220 & 620689 & CROC_1/AE.SQUARROSA (176)//KACHU/3/BAJ \#1 & $\begin{array}{l}\text { SDSS13Y00120T-0B- } \\
\text { 0Y-0M-0Y-0B-92Y }\end{array}$ & 6.17 & 4.17 & 1.70 & MS \\
\hline 275 & 620785 & IWA8614378/NAVJ07//KACHU & $\begin{array}{l}\text { SDSS13Y00216T-0B- } \\
\text { 0Y-0M-0Y-0B-123Y }\end{array}$ & 6.17 & 3.31 & 1.94 & MS \\
\hline 34 & 620594 & $\begin{array}{l}\text { INDIA-223/7/SHA7/VEE\#5/5/VEE\#8//JUP/BJY/3/ } \\
\text { F3.71/TRM/4/2*WEAVER/6/SKAUZ/PARUS//PARUS/ } \\
\text { 8/CNDO/R143//ENTE/MEXI_2/3/AEGILOPS } \\
\text { SQUARROSA (TAUS)/4/WEAVER/5/PICUS/6/ } \\
\text { TROST/7/TACUPETO F2001 }\end{array}$ & $\begin{array}{l}\text { SDSS13Y00025T-0B- } \\
\text { 0Y-0M-0Y-0B-27Y }\end{array}$ & 6.33 & 6.47 & 2.64 & MS \\
\hline 60 & 620620 & $\begin{array}{l}\text { IG 122193/4/PRL/2*PASTOR//PBW343*2/ } \\
\text { KUKUNA/3/ROLF07/5/NELOKI }\end{array}$ & $\begin{array}{l}\text { SDSS13Y00051T-0B- } \\
\text { 0Y-0M-0Y-0B-71Y }\end{array}$ & 6.33 & 4.23 & 1.73 & MS \\
\hline 78 & 620630 & H-1694/NELOKI/3/ATTILA*2/PBW65//MURGA & $\begin{array}{l}\text { SDSS13Y00061T-0B- } \\
\text { 0Y-0M-0Y-0B-48Y }\end{array}$ & 6.33 & 4.32 & 1.76 & MS \\
\hline 130 & 620641 & $\begin{array}{l}\text { 68.111/RGB-U//WARD RESEL/3/STIL/4/ } \\
\text { AE.SQUARROSA (630)/5/BORL14/6/COPIO }\end{array}$ & $\begin{array}{l}\text { SDSS13Y00072T-0B- } \\
\text { 0Y-0M-0Y-0B-62Y }\end{array}$ & 6.33 & 3.44 & 1.89 & MS \\
\hline 23 & 620583 & H-1624/BAJ \#1//SUP152 & $\begin{array}{l}\text { SDSS13Y00014T-0B- } \\
\text { 0Y-0M-0Y-0B-43Y }\end{array}$ & 6.50 & 4.55 & 2.69 & MS \\
\hline 64 & 620621 & $\begin{array}{l}\text { IG 122196/4/PRL/2*PASTOR//PBW343*2/ } \\
\text { KUKUNA/3/ROLF07/5/NELOKI }\end{array}$ & $\begin{array}{l}\text { SDSS13Y00052T-0B- } \\
\text { 0Y-0M-0Y-0B-88Y }\end{array}$ & 6.50 & 8.02 & 3.27 & MS \\
\hline 77 & 620630 & H-1694/NELOKI/3/ATTILA*2/PBW65//MURGA & $\begin{array}{l}\text { SDSS13Y00061T-0B- } \\
\text { 0Y-0M-0Y-0B-39Y }\end{array}$ & 6.50 & 4.51 & 1.84 & MS \\
\hline 155 & 620650 & IG 41620/COPIO/3/KACHU \#1/KIRITATI//KACHU & $\begin{array}{l}\text { SDSS13Y00081T-0B- } \\
\text { 0Y-0M-0Y-0B-64Y }\end{array}$ & 6.50 & 1.05 & 0.43 & MS \\
\hline 158 & 620652 & PERSIA-7/COPIO/3/KACHU \#1/KIRITATI//KACHU & $\begin{array}{l}\text { SDSS13Y00083T-0B- } \\
\text { 0Y-0M-0Y-0B-66Y }\end{array}$ & 6.50 & 4.51 & 2.40 & MS \\
\hline
\end{tabular}


Supplementary Table4(Continue): List of Drought and Heat Tolerant Spring Wheat lines and their Resistant Response to the CCN Heterodera filipjevi. (SD) Standard Deviation, (SE) Standard Error, (CID) Cross ID

\begin{tabular}{|c|c|c|c|c|c|c|c|}
\hline $\begin{array}{c}\text { ENTRY } \\
\text { NUMBER }\end{array}$ & $C I D$ & PEDIGREE (CROSS) & $\begin{array}{l}\text { SELECTION } \\
\text { HISTORY }\end{array}$ & $\begin{array}{c}\text { (MEAN) } \\
\text { CYSTS } \\
\text { AND } \\
\text { FEMALES }\end{array}$ & $S D$ & $S E$ & $\begin{array}{l}\text { RESISTANT } \\
\text { RESPONSE }\end{array}$ \\
\hline 274 & 620785 & IWA8614378/NAVJ07//KACHU & $\begin{array}{l}\text { SDSS13Y00216T-0B- } \\
\text { OY-0M-0Y-0B-121Y }\end{array}$ & 6.50 & 3.73 & 1.52 & MS \\
\hline 124 & 620641 & $\begin{array}{l}\text { 68.111/RGB-U//WARD RESEL/3/STIL/4/ } \\
\text { AE.SQUARROSA (630)/5/BORL14/6/COPIO }\end{array}$ & $\begin{array}{l}\text { SDSS13Y00072T-0B- } \\
\text { 0Y-0M-0Y-0B-51Y }\end{array}$ & 6.67 & 3.67 & 1.50 & MS \\
\hline 146 & 620647 & $\begin{array}{l}\text { T.DICOCCON PI94624/AE.SQUARROSA ( } 454) / / \\
\text { COPIO/3/KACHU \#1/KIRITATI//KACHU }\end{array}$ & $\begin{array}{l}\text { SDSS13Y00078T-0B- } \\
\text { 0Y-0M-0Y-0B-85Y }\end{array}$ & 6.67 & 3.72 & 2.17 & MS \\
\hline 211 & 620687 & CROC_1/AE.SQUARROSA (436)//KACHU/3/BAJ \#1 & $\begin{array}{l}\text { SDSS13Y00118T-0B- } \\
\text { 0Y-0M-0Y-0B-123Y }\end{array}$ & 6.67 & 6.65 & 2.72 & MS \\
\hline 249 & 620726 & $\begin{array}{l}\text { IG 107128/4/PRL/2*PASTOR//PBW343*2/KUKUNA } \\
\text { /3/ROLF07/5/NELOKI }\end{array}$ & $\begin{array}{l}\text { SDSS13Y00157T-0B- } \\
\text { 0Y-0M-0Y-0B-50Y }\end{array}$ & 6.67 & 8.89 & 3.63 & MS \\
\hline 277 & 620786 & $\begin{array}{l}\text { IWA8612701/6/KAUZ//ALTAR84/AOS/3/PASTOR/4/ } \\
\text { MILAN/CUPE//SW89.3064/5/KIRITATI/7/SW89.5277/ } \\
\text { BORL95//SKAUZ/3/PRL/2*PASTOR/4/HEILO }\end{array}$ & $\begin{array}{l}\text { SDSS13Y00217T-0B- } \\
\text { 0Y-0M-0Y-0B-63Y }\end{array}$ & 6.67 & 4.41 & 1.80 & MS \\
\hline 39 & 620595 & $\begin{array}{l}\text { CHIH95.4.6/7/SHA7/VEE\#5/5/VEE\#8//JUP/BJY/3/F3.7 } \\
\text { 1/TRM/4/2*WEAVER/6/SKAUZ/PARUS//PARUS/8/C } \\
\text { NDO/R143//ENTE/MEXI_2/3/AEGILOPS QUARROSA } \\
\text { (TAUS)/4/WEAVER/5/PICUS/6/TROST/7/TACUPETO } \\
\text { F2001 }\end{array}$ & $\begin{array}{l}\text { SDSS13Y00026T-0B- } \\
\text { 0Y-0M-0Y-0B-52Y }\end{array}$ & 6.83 & 7.55 & 3.08 & MS \\
\hline 82 & 620631 & H-1699/NELOKI/3/ATTILA*2/PBW65//MURGA & $\begin{array}{l}\text { SDSS13Y00062T-0B- } \\
\text { 0Y-0M-0Y-0B-26Y }\end{array}$ & 6.83 & 4.40 & 2.14 & MS \\
\hline 109 & 620638 & $\begin{array}{l}\text { LOCAL RED/AE.SQUARROSA } \\
(223) / / \text { BORL14/3/COPIO }\end{array}$ & $\begin{array}{l}\text { SDSS13Y00069T-0B- } \\
\text { 0Y-0M-0Y-0B-56Y }\end{array}$ & 6.83 & 7.33 & 2.99 & MS \\
\hline 139 & 620645 & IWA8612416/BORL14//COPIO & $\begin{array}{l}\text { SDSS13Y00076T-0B- } \\
\text { 0Y-0M-0Y-0B-49Y }\end{array}$ & 6.83 & 3.87 & 2.21 & MS \\
\hline 217 & 620687 & CROC_1/AE.SQUARROSA (436)//KACHU/3/BAJ \#1 & $\begin{array}{l}\text { SDSS13Y00118T-0B- } \\
\text { 0Y-0M-0Y-0B-145Y }\end{array}$ & 6.83 & 3.43 & 1.40 & MS \\
\hline 236 & 620698 & IG 41474/NAVJ07//KACHU & $\begin{array}{l}\text { SDSS13Y00129T-0B- } \\
\text { 0Y-0M-0Y-0B-46Y }\end{array}$ & 6.83 & 4.31 & 2.14 & MS \\
\hline 253 & 620736 & $\begin{array}{l}\text { IG 122627/6/KAUZ//ALTAR 84/AOS/3/PASTOR/4/ } \\
\text { MILAN/CUPE//SW89.3064/5/KIRITATI/7/SW89.5277/ } \\
\text { BORL95//SKAUZ/3/PRL/2*PASTOR/4/HEILO }\end{array}$ & $\begin{array}{l}\text { SDSS13Y00167T-0B- } \\
\text { 0Y-0M-0Y-0B-37Y }\end{array}$ & 6.83 & 5.04 & 2.06 & MS \\
\hline 18 & 620583 & H-1624/BAJ \#1//SUP152 & $\begin{array}{l}\text { SDSS13Y00014T-0B- } \\
\text { 0Y-0M-0Y-0B-29Y }\end{array}$ & 7.00 & 3.69 & 2.11 & MS \\
\hline 45 & 620600 & $\begin{array}{l}\text { H-1357/8/CNDO/R143//ENTE/MEXI_2/3/AEGILOPS } \\
\text { SQUARROSA (TAUS)/4/WEAVER/5/PICUS/6/TROST } \\
\text { /7/TACUPETO F2001/9/KAUZ//ALTAR 84/AOS/3/ } \\
\text { PASTOR/4/MILAN/CUPE//SW89.3064/5/KIRITATI }\end{array}$ & $\begin{array}{l}\text { SDSS13Y00031T-0B- } \\
\text { 0Y-0M-0Y-0B-50Y }\end{array}$ & 7.00 & 4.69 & 2.38 & MS \\
\hline 51 & 620612 & $\begin{array}{l}\text { IG 41489/4/PRL/2*PASTOR//PBW343*2/ } \\
\text { KUKUNA/3/ROLF07/5/NELOKI }\end{array}$ & $\begin{array}{l}\text { SDSS13Y00043T-0B- } \\
\text { 0Y-0M-0Y-0B-40Y }\end{array}$ & 7.00 & 4.52 & 1.84 & MS \\
\hline 52 & 620612 & $\begin{array}{l}\text { IG 41489/4/PRL/2*PASTOR//PBW343*2/ } \\
\text { KUKUNA/3/ROLF07/5/NELOKI }\end{array}$ & $\begin{array}{l}\text { SDSS13Y00043T-0B- } \\
\text { 0Y-0M-0Y-0B-54Y }\end{array}$ & 7.00 & 2.97 & 2.50 & MS \\
\hline 55 & 620613 & $\begin{array}{l}\text { IG 41505/4/PRL/2*PASTOR//PBW343*2/ } \\
\text { KUKUNA/3/ROLF07/5/NELOKI }\end{array}$ & $\begin{array}{l}\text { SDSS13Y00044T-0B- } \\
\text { 0Y-0M-0Y-0B-34Y }\end{array}$ & 7.00 & 4.82 & 3.40 & MS \\
\hline 56 & 620615 & $\begin{array}{l}\text { IG 122145/4/PRL/2*PASTOR//PBW343*2/ } \\
\text { KUKUNA/3/ROLF07/5/NELOKI }\end{array}$ & $\begin{array}{l}\text { SDSS13Y00046T-0B- } \\
\text { 0Y-0M-0Y-0B-36Y }\end{array}$ & 7.00 & 4.00 & 1.63 & MS \\
\hline 62 & 620620 & $\begin{array}{l}\text { IG 122193/4/PRL/2*PASTOR//PBW343*2/ } \\
\text { KUKUNA/3/ROLF07/5/NELOKI }\end{array}$ & $\begin{array}{l}\text { SDSS13Y00051T-0B- } \\
\text { 0Y-0M-0Y-0B-75Y }\end{array}$ & 7.00 & 3.10 & 1.26 & MS \\
\hline 97 & 620635 & INDIA-50/3/ATTILA*2/PBW65//MURGA/4/BORL14 & $\begin{array}{l}\text { SDSS13Y00066T-0B- } \\
\text { 0Y-0M-0Y-0B-60Y }\end{array}$ & 7.00 & 3.22 & 1.32 & MS \\
\hline 84 & 620631 & H-1699/NELOKI/3/ATTILA*2/PBW65//MURGA & $\begin{array}{l}\text { SDSS13Y00062T-0B- } \\
\text { 0Y-0M-0Y-0B-30Y }\end{array}$ & 7.17 & 4.45 & 1.82 & MS \\
\hline 90 & 620634 & INDIA-38/3/ATTILA*2/PBW65//MURGA/4/BORL14 & $\begin{array}{l}\text { SDSS13Y00065T-0B- } \\
\text { 0Y-0M-0Y-0B-48Y }\end{array}$ & 7.17 & 7.36 & 3.00 & MS \\
\hline 98 & 620635 & INDIA-50/3/ATTILA*2/PBW65//MURGA/4/BORL14 & $\begin{array}{l}\text { SDSS13Y00066T-0B- } \\
\text { 0Y-0M-0Y-0B-61Y }\end{array}$ & 7.17 & 4.62 & 2.18 & MS \\
\hline 106 & 620638 & $\begin{array}{l}\text { LOCAL RED/AE.SQUARROSA (223)//BORL14/3/ } \\
\text { COPIO }\end{array}$ & $\begin{array}{l}\text { SDSS13Y00069T-0B- } \\
\text { 0Y-0M-0Y-0B-40Y }\end{array}$ & 7.17 & 3.87 & 1.58 & MS \\
\hline 197 & 620678 & H-1491/ROLF07//NAVJ07 & $\begin{array}{l}\text { SDSS13Y00109T-0B- } \\
\text { 0Y-0M-0Y-0B-73Y }\end{array}$ & 7.17 & 2.32 & 0.95 & MS \\
\hline 245 & 620710 & IG 41735/NAVJ07//KACHU & $\begin{array}{l}\text { SDSS13Y00141T-0B- } \\
\text { 0Y-0M-0Y-0B-68Y }\end{array}$ & 7.17 & 8.45 & 3.45 & MS \\
\hline 255 & 620736 & $\begin{array}{l}\text { IG 122627/6/KAUZ//ALTAR 84/AOS/3/PASTOR/4/ } \\
\text { MILAN/CUPE//SW89.3064/5/KIRITATI/7/SW89.5277/ } \\
\text { BORL95//SKAUZ/3/PRL/2*PASTOR/4/HEILO }\end{array}$ & $\begin{array}{l}\text { SDSS13Y00167T-0B- } \\
\text { 0Y-0M-0Y-0B-41Y }\end{array}$ & 7.17 & 3.31 & 1.89 & MS \\
\hline 74 & 620629 & H-1546/NELOKI/3/ATTILA*2/PBW65//MURGA & $\begin{array}{l}\text { SDSS13Y00060T-0B- } \\
\text { 0Y-0M-0Y-0B-49Y }\end{array}$ & 7.33 & 4.89 & 2.68 & MS \\
\hline 83 & 620631 & H-1699/NELOKI/3/ATTILA*2/PBW65//MURGA & $\begin{array}{l}\text { SDSS13Y00062T-0B- } \\
\text { 0Y-0M-0Y-0B-28Y }\end{array}$ & 7.33 & 3.61 & 1.48 & MS \\
\hline 154 & 620648 & $\begin{array}{l}\text { T.DICOCCON PI94625/AE.SQUARROSA (372)// } \\
\text { COPIO/3/KACHU \#1/KIRITATI//KACHU }\end{array}$ & $\begin{array}{l}\text { SDSS13Y00079T-0B- } \\
\text { 0Y-0M-0Y-0B-113Y }\end{array}$ & 7.33 & 4.50 & 2.33 & MS \\
\hline
\end{tabular}


Supplementary Table5(Continue): List of Drought and Heat Tolerant Spring Wheat lines and their Resistant Response to the CCN Heterodera filipjevi. (SD) Standard Deviation, (SE) Standard Error, (CID) Cross ID

\begin{tabular}{|c|c|c|c|c|c|c|c|}
\hline $\begin{array}{c}\text { ENTRY } \\
\text { NUMBER }\end{array}$ & $C I D$ & PEDIGREE (CROSS) & $\begin{array}{l}\text { SELECTION } \\
\text { HISTORY }\end{array}$ & $\begin{array}{l}\text { (MEAN) } \\
\text { CYSTS } \\
\text { AND } \\
\text { FEMALES }\end{array}$ & $S D$ & $S E$ & $\begin{array}{l}\text { RESISTANT } \\
\text { RESPONSE }\end{array}$ \\
\hline 267 & 620768 & $\begin{array}{l}\text { H-1659/3/PBW343*2/KUKUNA*2//FRTL/PIFED/4/ } \\
\text { QUAIU \#1 }\end{array}$ & $\begin{array}{l}\text { SDSS13Y00199T-0B- } \\
\text { 0Y-0M-0Y-0B-39Y }\end{array}$ & 7.33 & 3.14 & 1.28 & MS \\
\hline 131 & 620642 & $\begin{array}{l}\text { D67.2/PARANA 66.270//AE.SQUARROSA (1085)/3/ } \\
\text { BORL14/4/COPIO }\end{array}$ & $\begin{array}{l}\text { SDSS13Y00073T-0B- } \\
\text { 0Y-0M-0Y-0B-73Y }\end{array}$ & 7.50 & 5.24 & 2.14 & $\mathrm{~S}$ \\
\hline 136 & 620645 & IWA8612416/BORL14//COPIO & $\begin{array}{l}\text { SDSS13Y00076T-0B- } \\
\text { 0Y-0M-0Y-0B-40Y }\end{array}$ & 7.50 & 4.81 & 1.96 & $\mathrm{~S}$ \\
\hline 205 & 620684 & MEX94.30.10/NAVJ07//KACHU & $\begin{array}{l}\text { SDSS13Y00115T-0B- } \\
\text { 0Y-0M-0Y-0B-102Y }\end{array}$ & 7.50 & 3.78 & 1.54 & $\mathrm{~S}$ \\
\hline 223 & 620690 & GAN/AE.SQUARROSA (206)//KACHU/3/BAJ \#1 & $\begin{array}{l}\text { SDSS13Y00121T-0B- } \\
\text { 0Y-0M-0Y-0B-59Y }\end{array}$ & 7.50 & 3.39 & 1.38 & $\mathrm{~S}$ \\
\hline 226 & 620692 & $\begin{array}{l}\text { D67.2/PARANA 66.270//AE.SQUARROSA (448)/3/ } \\
\text { KACHU/4/BAJ \#1 }\end{array}$ & $\begin{array}{l}\text { SDSS13Y00123T-0B- } \\
\text { 0Y-0M-0Y-0B-122Y }\end{array}$ & 7.50 & 2.59 & 1.06 & $\mathrm{~S}$ \\
\hline 243 & 620699 & $\begin{array}{l}\text { IG 41506/3/PBW343*2/KUKUNA*2//FRTL/ } \\
\text { PIFED/4/QUAIU \#1 }\end{array}$ & $\begin{array}{l}\text { SDSS13Y00130T-0B- } \\
\text { 0Y-0M-0Y-0B-83Y }\end{array}$ & 7.50 & 4.76 & 1.95 & $\mathrm{~S}$ \\
\hline $\begin{array}{l}\text { BEZOST } \\
\text { AJA (S) }\end{array}$ & & LUT17/SRS2 & & 7.50 & 2.65 & 1.32 & $\mathrm{~S}$ \\
\hline 37 & 620595 & $\begin{array}{l}\text { CHIH95.4.6/7/SHA7/VEE\#5/5/VEE\#8//JUP/BJY/3/F3.7 } \\
\text { 1/TRM/4/2*WEAVER/6/SKAUZ/PARUS//PARUS/8/C } \\
\text { NDO/R143//ENTE/MEXI_2/3/AEGILOPS QUARROSA } \\
\text { (TAUS)/4/WEAVER/5/PICUS/6/TROST/7/TACUPETO } \\
\text { F2001 }\end{array}$ & $\begin{array}{l}\text { SDSS13Y00026T-0B- } \\
\text { 0Y-0M-0Y-0B-24Y }\end{array}$ & 7.67 & 2.80 & 1.15 & $\mathrm{~S}$ \\
\hline 38 & 620595 & $\begin{array}{l}\text { CHIH95.4.6/7/SHA7/VEE\#5/5/VEE\#8//JUP/BJY/3/F3.7 } \\
\text { 1/TRM/4/2*WEAVER/6/SKAUZ/PARUS//PARUS/8/C } \\
\text { NDO/R143//ENTE/MEXI_2/3/AEGILOPS QUARROSA } \\
\text { (TAUS)/4/WEAVER/5/PICUS/6/TROST/7/TACUPETO } \\
\text { F2001 }\end{array}$ & $\begin{array}{l}\text { SDSS13Y00026T-0B- } \\
\text { 0Y-0M-0Y-0B-34Y }\end{array}$ & 7.67 & 4.32 & 1.76 & $\mathrm{~S}$ \\
\hline 119 & 620641 & $\begin{array}{l}\text { 68.111/RGB-U//WARD } \\
\text { RESEL/3/STIL/4/AE.SQUARROSA } \\
\text { (630)/5/BORL14/6/COPIO }\end{array}$ & $\begin{array}{l}\text { SDSS13Y00072T-0B- } \\
\text { 0Y-0M-0Y-0B-43Y }\end{array}$ & 7.67 & 8.87 & 3.62 & $\mathrm{~S}$ \\
\hline 201 & 620683 & H-1601/NAVJ07//KACHU & $\begin{array}{l}\text { SDSS13Y00114T-0B- } \\
\text { 0Y-0M-0Y-0B-100Y }\end{array}$ & 7.67 & 5.13 & 3.12 & $\mathrm{~S}$ \\
\hline 231 & 620695 & INDIA-107/KACHU//BAJ \#1 & $\begin{array}{l}\text { SDSS13Y00126T-0B- } \\
\text { 0Y-0M-0Y-0B-87Y }\end{array}$ & 7.67 & 4.23 & 1.73 & $S$ \\
\hline 4 & 620575 & DOY1/AE.SQUARROSA (488)//BAJ \#1/3/SUP152 & $\begin{array}{l}\text { SDSS13Y00006T-0B- } \\
\text { 0Y-0M-0Y-0B-57Y }\end{array}$ & 7.83 & 4.26 & 1.74 & $\mathrm{~S}$ \\
\hline 9 & 620577 & DVERD_2/AE.SQUARROSA (333)//BAJ \#1/3/SUP152 & $\begin{array}{l}\text { SDSS13Y00008T-0B- } \\
\text { 0Y-0M-0Y-0B-55Y }\end{array}$ & 7.83 & 8.16 & 3.33 & $\mathrm{~S}$ \\
\hline 11 & 620577 & DVERD_2/AE.SQUARROSA (333)//BAJ \#1/3/SUP152 & $\begin{array}{l}\text { SDSS13Y00008T-0B- } \\
\text { 0Y-0M-0Y-0B-61Y }\end{array}$ & 7.83 & 3.76 & 1.54 & $\mathrm{~S}$ \\
\hline 149 & 620648 & $\begin{array}{l}\text { T.DICOCCON PI94625/AE.SQUARROSA (372)// } \\
\text { COPIO/3/KACHU \#1/KIRITATI//KACHU }\end{array}$ & $\begin{array}{l}\text { SDSS13Y00079T-0B- } \\
\text { 0Y-0M-0Y-0B-105Y }\end{array}$ & 7.83 & 3.54 & 1.45 & $\mathrm{~S}$ \\
\hline 182 & 620661 & $\begin{array}{l}\text { YAV79//DACK/RABI/3/SNIPE/4/AE.SQUARROSA } \\
(460) / 5 / \mathrm{KACHU} \# 1 / \mathrm{KIRITATI} / \mathrm{KACHU} / 6 / \mathrm{PBW} 343 * 2 / \\
\text { KUKUNA*2//FRTL/PIFED }\end{array}$ & $\begin{array}{l}\text { SDSS13Y00092T-0B- } \\
\text { 0Y-0M-0Y-0B-100Y }\end{array}$ & 7.83 & 5.38 & 2.20 & $S$ \\
\hline 212 & 620687 & CROC_1/AE.SQUARROSA (436)//KACHU/3/BAJ \#1 & $\begin{array}{l}\text { SDSS13Y00118T-0B- } \\
\text { 0Y-0M-0Y-0B-124Y }\end{array}$ & 7.83 & 2.40 & 0.98 & $\mathrm{~S}$ \\
\hline 228 & 620694 & INDIA-59/KACHU//BAJ \#1 & $\begin{array}{l}\text { SDSS13Y00125T-0B- } \\
\text { 0Y-0M-0Y-0B-73Y }\end{array}$ & 7.83 & 5.04 & 2.80 & $\mathrm{~S}$ \\
\hline 242 & 620699 & $\begin{array}{l}\text { IG 41506/3/PBW343*2/KUKUNA*2//FRTL/ } \\
\text { PIFED/4/QUAIU \#1 }\end{array}$ & $\begin{array}{l}\text { SDSS13Y00130T-0B- } \\
\text { 0Y-0M-0Y-0B-78Y }\end{array}$ & 7.83 & 5.04 & 2.06 & $\mathrm{~S}$ \\
\hline 31 & 620588 & $\begin{array}{l}\text { H-1311/3/FRET2*2/SHAMA//KACHU/4/ } \\
\text { HUW234+LR34/PRINIA*2//KIRITATI }\end{array}$ & $\begin{array}{l}\text { SDSS13Y00019T-0B- } \\
\text { 0Y-0M-0Y-0B-16Y }\end{array}$ & 8.00 & 3.29 & 1.34 & $\mathrm{~S}$ \\
\hline 40 & 620598 & $\begin{array}{l}\text { IG 122727/8/CNDO/R143//ENTE/MEXI_2/3/ } \\
\text { AEGILOPS SQUARROSA (TAUS)/4/WEAVER/5/ } \\
\text { PICUS/6/TROST/7/TACUPETO } \\
\text { 2001/9/KAUZ//ALTAR } \\
\text { 84/AOS/3/PASTOR/4/MILAN/CUPE//SW89.3064/5/KI } \\
\text { RITATI }\end{array}$ & $\begin{array}{l}\text { SDSS13Y00029T-0B- } \\
\text { 0Y-0M-0Y-0B-55Y }\end{array}$ & 8.00 & 4.86 & 1.98 & $\mathrm{~S}$ \\
\hline 76 & 620630 & H-1694/NELOKI/3/ATTILA*2/PBW65//MURGA & $\begin{array}{l}\text { SDSS13Y00061T-0B- } \\
\text { 0Y-0M-0Y-0B-36Y }\end{array}$ & 8.00 & 10.79 & 4.40 & $\mathrm{~S}$ \\
\hline 150 & 620648 & $\begin{array}{l}\text { T.DICOCCON PI94625/AE.SQUARROSA (372)// } \\
\text { COPIO/3/KACHU \#1/KIRITATI//KACHU }\end{array}$ & $\begin{array}{l}\text { SDSS13Y00079T-0B- } \\
\text { 0Y-0M-0Y-0B-106Y }\end{array}$ & 8.00 & 5.06 & 2.70 & $\mathrm{~S}$ \\
\hline 178 & 620657 & $\begin{array}{l}\text { CETA/AE.SQUARROSA ( } 895) / 3 / \text { KACHU \#1/ } \\
\text { KIRITATI//KACHU/4/PBW343*2/KUKUNA*2//FRTL/ } \\
\text { PIFED }\end{array}$ & $\begin{array}{l}\text { SDSS13Y00088T-0B- } \\
\text { 0Y-0M-0Y-0B-120Y }\end{array}$ & 8.00 & 5.37 & 3.59 & $S$ \\
\hline 196 & 620678 & H-1491/ROLF07//NAVJ07 & $\begin{array}{l}\text { SDSS13Y00109T-0B- } \\
\text { 0Y-0M-0Y-0B-54Y }\end{array}$ & 8.00 & 4.60 & 1.88 & $\mathrm{~S}$ \\
\hline 27 & 620586 & $\begin{array}{l}\text { INDIA-101/3/FRET2*2/SHAMA//KACHU/4/ } \\
\text { HUW234+LR34/PRINIA*2//KIRITATI }\end{array}$ & $\begin{array}{l}\text { SDSS13Y00017T-0B- } \\
\text { 0Y-0M-0Y-0B-61Y }\end{array}$ & 8.17 & 5.49 & 2.24 & $\mathrm{~S}$ \\
\hline 58 & 620616 & $\begin{array}{l}\text { IG } \\
\text { 122146/4/PRL/2*PASTOR//PBW343*2/KUKUNA/3/R } \\
\text { OLF07/5/NELOKI }\end{array}$ & $\begin{array}{l}\text { SDSS13Y00047T-0B- } \\
\text { 0Y-0M-0Y-0B-71Y }\end{array}$ & 8.17 & 4.71 & 3.03 & $\mathrm{~S}$ \\
\hline 108 & 620638 & $\begin{array}{l}\text { LOCAL RED/AE.SQUARROSA (223)// } \\
\text { BORL14/3/COPIO }\end{array}$ & $\begin{array}{l}\text { SDSS13Y00069T-0B- } \\
\text { 0Y-0M-0Y-0B-55Y }\end{array}$ & 8.17 & 5.53 & 3.12 & $\mathrm{~S}$ \\
\hline
\end{tabular}


Supplementary Table6(Continue): List of Drought and Heat Tolerant Spring Wheat lines and their Resistant Response to the CCN Heterodera filipjevi. (SD) Standard Deviation, (SE) Standard Error, (CID) Cross ID

\begin{tabular}{|c|c|c|c|c|c|c|c|}
\hline $\begin{array}{c}\text { ENTRY } \\
\text { NUMBER }\end{array}$ & $C I D$ & PEDIGREE (CROSS) & $\begin{array}{l}\text { SELECTION } \\
\text { HISTORY }\end{array}$ & $\begin{array}{c}\text { (MEAN) } \\
\text { CYSTS } \\
\text { AND } \\
\text { FEMALES }\end{array}$ & $S D$ & $S E$ & $\begin{array}{l}\text { RESISTANT } \\
\text { RESPONSE }\end{array}$ \\
\hline 200 & 620680 & IG 41243/NAVJ07//KACHU & $\begin{array}{l}\text { SDSS13Y00111T-0B- } \\
\text { 0Y-0M-0Y-0B-64Y }\end{array}$ & 8.17 & 5.71 & 3.24 & S \\
\hline 207 & 620685 & ARLIN/AE.SQUARROSA (283)//KACHU/3/BAJ \#1 & $\begin{array}{l}\text { SDSS13Y00116T-0B- } \\
\text { 0Y-0M-0Y-0B-83Y }\end{array}$ & 8.17 & 5.64 & 2.30 & $\mathrm{~S}$ \\
\hline 209 & 620687 & CROC_1/AE.SQUARROSA (436)//KACHU/3/BAJ \#1 & $\begin{array}{l}\text { SDSS13Y00118T-0B- } \\
\text { 0Y-0M-0Y-0B-111Y }\end{array}$ & 8.17 & 5.04 & 3.07 & S \\
\hline 240 & 620699 & $\begin{array}{l}\text { IG } \\
41506 / 3 / \mathrm{PBW} 343 * 2 / \mathrm{KUKUNA} * 2 / / \mathrm{FRTL} / \mathrm{PIFED} / 4 / \mathrm{QUA} \\
\text { IU \#1 }\end{array}$ & $\begin{array}{l}\text { SDSS13Y00130T-0B- } \\
\text { 0Y-0M-0Y-0B-74Y }\end{array}$ & 8.17 & 3.37 & 1.38 & $\mathrm{~S}$ \\
\hline 2 & 620575 & DOY1/AE.SQUARROSA (488)//BAJ \#1/3/SUP152 & $\begin{array}{l}\text { SDSS13Y00006T-0B- } \\
\text { 0Y-0M-0Y-0B-52Y }\end{array}$ & 8.33 & 3.08 & 3.09 & S \\
\hline 48 & 620604 & $\begin{array}{l}\text { IG 42152/6/KAUZ//ALTAR 84/AOS/3/PASTOR/4/ } \\
\text { MILAN/CUPE//SW89.3064/5/KIRITAT//7/SW89.5277/ } \\
\text { BORL95//SKAUZ/3/PRL/2*PASTOR/4/HEILO }\end{array}$ & $\begin{array}{l}\text { SDSS13Y00035T-0B- } \\
\text { 0Y-0M-0Y-0B-60Y }\end{array}$ & 8.33 & 4.89 & 1.99 & $\mathrm{~S}$ \\
\hline 102 & 620635 & INDIA-50/3/ATTILA*2/PBW65//MURGA/4/BORL14 & $\begin{array}{l}\text { SDSS13Y00066T-0B- } \\
\text { 0Y-0M-0Y-0B-67Y }\end{array}$ & 8.33 & 5.65 & 2.65 & $\mathrm{~S}$ \\
\hline 118 & 620641 & $\begin{array}{l}\text { 68.111/RGB-U//WARD RESEL/3/STIL/4/ } \\
\text { AE.SQUARROSA (630)/5/BORL14/6/COPIO }\end{array}$ & $\begin{array}{l}\text { SDSS13Y00072T-0B- } \\
\text { 0Y-0M-0Y-0B-42Y }\end{array}$ & 8.33 & 4.68 & 1.91 & $\mathrm{~S}$ \\
\hline 16 & 620580 & $\begin{array}{l}\text { GARZA/BOY//AE.SQUARROSA (695)/3/BAJ \#1/4/ } \\
\text { SUP152 }\end{array}$ & $\begin{array}{l}\text { SDSS13Y00011T-0B- } \\
\text { 0Y-0M-0Y-0B-44Y }\end{array}$ & 8.50 & 5.32 & 2.17 & $\mathrm{~S}$ \\
\hline 32 & 620592 & $\begin{array}{l}\text { 68.111/RGB-U//WARD } \\
\text { RESEL/3/STIL/4/AE.SQUARROSA } \\
\text { (390)/7/SHA7/VEE\#5/5/VEE\#8//JUP/BJY/3/F3.71/TRM } \\
\text { /4/2*WEAVER/6/SKAUZ/PARUS//PARUS/8/CNDO/R } \\
\text { 143//ENTE/MEXI_2/3/AEGILOPS SQUARROSA } \\
\text { (TAUS)/4/WEAVER/5/PICUS/6/TROST/7/TACUPETO } \\
\text { F2001 }\end{array}$ & $\begin{array}{l}\text { SDSS13Y00023T-0B- } \\
\text { 0Y-0M-0Y-0B-60Y }\end{array}$ & 8.50 & 5.09 & 2.08 & $\mathrm{~S}$ \\
\hline 53 & 620613 & $\begin{array}{l}\text { IG 41505/4/PRL/2*PASTOR//PBW343*2/ } \\
\text { KUKUNA/3/ROLF07/5/NELOKI }\end{array}$ & $\begin{array}{l}\text { SDSS13Y00044T-0B- } \\
\text { 0Y-0M-0Y-0B-28Y }\end{array}$ & 8.50 & 5.32 & 3.00 & $\mathrm{~S}$ \\
\hline 113 & 620640 & $\begin{array}{l}\text { 68.111/RGB-U//WARD } \\
\text { RESEL/3/STIL/4/AE.SQUARROSA } \\
\text { (628)/5/BORL14/6/COPIO }\end{array}$ & $\begin{array}{l}\text { SDSS13Y00071T-0B- } \\
\text { 0Y-0M-0Y-0B-45Y }\end{array}$ & 8.50 & 9.03 & 3.69 & $\mathrm{~S}$ \\
\hline 134 & 620642 & $\begin{array}{l}\text { D67.2/PARANA 66.270//AE.SQUARROSA (1085) } \\
\text { /3/BORL14/4/COPIO }\end{array}$ & $\begin{array}{l}\text { SDSS13Y00073T-0B- } \\
\text { 0Y-0M-0Y-0B-80Y }\end{array}$ & 8.50 & 5.92 & 3.29 & $\mathrm{~S}$ \\
\hline 162 & 620652 & PERSIA-7/COPIO/3/KACHU \#1/KIRITATI//KACHU & $\begin{array}{l}\text { SDSS13Y00083T-0B- } \\
\text { 0Y-0M-0Y-0B-73Y }\end{array}$ & 8.50 & 4.55 & 2.46 & $\mathrm{~S}$ \\
\hline 167 & 620653 & PERSIA-21/COPIO/3/KACHU \#1/KIRITATI//KACHU & $\begin{array}{l}\text { SDSS13Y00084T-0B- } \\
\text { 0Y-0M-0Y-0B-74Y }\end{array}$ & 8.50 & 5.89 & 2.40 & $\mathrm{~S}$ \\
\hline 224 & 620690 & GAN/AE.SQUARROSA (206)//KACHU/3/BAJ \#1 & $\begin{array}{l}\text { SDSS13Y00121T-0B- } \\
\text { 0Y-0M-0Y-0B-79Y }\end{array}$ & 8.50 & 5.68 & 2.72 & $\mathrm{~S}$ \\
\hline 142 & 620646 & IWA8611400/BORL14//COPIO & $\begin{array}{l}\text { SDSS13Y00077T-0B- } \\
\text { 0Y-0M-0Y-0B-106Y }\end{array}$ & 8.67 & 5.43 & 2.78 & $\mathrm{~S}$ \\
\hline 269 & 620768 & $\begin{array}{l}\text { H-1659/3/PBW343*2/KUKUNA*2//FRTL/PIFED } \\
\text { /4/QUAIU \#1 }\end{array}$ & $\begin{array}{l}\text { SDSS13Y00199T-0B- } \\
\text { 0Y-0M-0Y-0B-44Y }\end{array}$ & 8.67 & 5.50 & 3.07 & $\mathrm{~S}$ \\
\hline 22 & 620583 & H-1624/BAJ \#1//SUP152 & $\begin{array}{l}\text { SDSS13Y00014T-0B- } \\
\text { 0Y-0M-0Y-0B-37Y }\end{array}$ & 8.83 & 5.12 & 2.52 & $\mathrm{~S}$ \\
\hline 36 & 620594 & $\begin{array}{l}\text { INDIA-223/7/SHA7/VEE\#5/5/VEE\#8//JUP/BJY/3/ } \\
\text { F3.71/TRM/4/2*WEAVER/6/SKAUZ/PARUS//PARUS/ } \\
\text { 8/CNDO/R143//ENTE/MEXI_2/3/AEGILOPS } \\
\text { SQUARROSA (TAUS)/4/WEAVER/5/PICUS/6/ } \\
\text { TROST/7/TACUPETO F2001 }\end{array}$ & $\begin{array}{l}\text { SDSS13Y00025T-0B- } \\
\text { 0Y-0M-0Y-0B-48Y }\end{array}$ & 8.83 & 5.64 & 2.77 & $\mathrm{~S}$ \\
\hline 59 & 620616 & $\begin{array}{l}\text { IG 122146/4/PRL/2*PASTOR//PBW343*2/ } \\
\text { KUKUNA/3/ROLF07/5/NELOKI }\end{array}$ & $\begin{array}{l}\text { SDSS13Y00047T-0B- } \\
\text { 0Y-0M-0Y-0B-74Y }\end{array}$ & 8.83 & 5.38 & 3.29 & $S$ \\
\hline 75 & 620629 & H-1546/NELOKI/3/ATTILA*2/PBW65//MURGA & $\begin{array}{l}\text { SDSS13Y00060T-0B- } \\
\text { 0Y-0M-0Y-0B-51Y }\end{array}$ & 8.83 & 5.04 & 2.06 & $\mathrm{~S}$ \\
\hline 115 & 620640 & $\begin{array}{l}\text { 68.111/RGB-U//WARD RESEL/3/STIL/4/ } \\
\text { AE.SQUARROSA (628)/5/BORL14/6/COPIO }\end{array}$ & $\begin{array}{l}\text { SDSS13Y00071T-0B- } \\
\text { 0Y-0M-0Y-0B-47Y }\end{array}$ & 8.83 & 5.71 & 2.33 & S \\
\hline 135 & 620645 & IWA8612416/BORL14//COPIO & $\begin{array}{l}\text { SDSS13Y00076T-0B- } \\
\text { 0Y-0M-0Y-0B-35Y }\end{array}$ & 8.83 & 5.81 & 2.94 & $\mathrm{~S}$ \\
\hline 252 & 620736 & $\begin{array}{l}\text { IG 122627/6/KAUZ//ALTAR 84/AOS/3/PASTOR } \\
\text { /4/MILAN/CUPE//SW89.3064/5/KIRITATI/7/SW89.527 } \\
\text { 7/BORL95//SKAUZ/3/PRL/2*PASTOR/4/HEILO }\end{array}$ & $\begin{array}{l}\text { SDSS13Y00167T-0B- } \\
\text { 0Y-0M-0Y-0B-34Y }\end{array}$ & 8.83 & 5.85 & 2.99 & $\mathrm{~S}$ \\
\hline 17 & 620581 & IG 42134/BAJ \#1//SUP152 & $\begin{array}{l}\text { SDSS13Y00012T-0B- } \\
\text { 0Y-0M-0Y-0B-54Y }\end{array}$ & 9.00 & 5.40 & 2.70 & $S$ \\
\hline 129 & 620641 & $\begin{array}{l}\text { 68.111/RGB-U//WARD RESEL/3/STIL/4/ } \\
\text { AE.SQUARROSA (630)/5/BORL14/6/COPIO }\end{array}$ & $\begin{array}{l}\text { SDSS13Y00072T-0B- } \\
\text { 0Y-0M-0Y-0B-60Y }\end{array}$ & 9.00 & 5.90 & 3.34 & S \\
\hline 171 & 620654 & $\begin{array}{l}\text { CETA/AE.SQUARROSA (850)/3/KACHU \#1/ } \\
\text { KIRITATI/KACHU/4/PBW343*2/KUKUNA*2//FRTL/ } \\
\text { PIFED }\end{array}$ & $\begin{array}{l}\text { SDSS13Y00085T-0B- } \\
\text { 0Y-0M-0Y-0B-62Y }\end{array}$ & 9.00 & 5.55 & 2.27 & $\mathrm{~S}$ \\
\hline 183 & 620662 & $\begin{array}{l}\text { YAV79//DACK/RABI/3/SNIPE/4/AE.SQUARROSA } \\
\text { (477)/5/KACHU \#1/KIRITATI//KACHU/6/ } \\
\text { PBW343*2/KUKUNA*2//FRTL/PIFED }\end{array}$ & $\begin{array}{l}\text { SDSS13Y00093T-0B- } \\
\text { 0Y-0M-0Y-0B-82Y }\end{array}$ & 9.00 & 5.51 & 2.68 & $\mathrm{~S}$ \\
\hline
\end{tabular}


Supplementary Table7(Continue): List of Drought and Heat Tolerant Spring Wheat lines and their Resistant Response to the CCN Heterodera filipjevi. (SD) Standard Deviation, (SE) Standard Error, (CID) Cross ID

\begin{tabular}{|c|c|c|c|c|c|c|c|}
\hline $\begin{array}{l}\text { ENTRY } \\
\text { NUMBER }\end{array}$ & $C I D$ & PEDIGREE (CROSS) & $\begin{array}{l}\text { SELECTION } \\
\text { HISTORY }\end{array}$ & $\begin{array}{l}\text { (MEAN) } \\
\text { CYSTS } \\
\text { AND } \\
\text { FEMALES }\end{array}$ & $S D$ & $S E$ & $\begin{array}{l}\text { RESISTANT } \\
\text { RESPONSE }\end{array}$ \\
\hline 276 & 620785 & IWA8614378/NAVJ07//KACHU & $\begin{array}{l}\text { SDSS13Y00216T-0B- } \\
\text { 0Y-0M-0Y-0B-125Y }\end{array}$ & 9.00 & 5.93 & 2.42 & $\mathrm{~S}$ \\
\hline 43 & 620600 & $\begin{array}{l}\text { H-1357/8/CNDO/R143//ENTE/MEXI_2/3/AEGILOPS } \\
\text { SQUARROSA (TAUS)/4/WEAVER/5/PICUS/6/TROST } \\
\text { /7/TACUPETO F2001/9/KAUZ//ALTAR 84/AOS/3/ } \\
\text { PASTOR/4/MILAN/CUPE//SW89.3064/5/KIRITATI }\end{array}$ & $\begin{array}{l}\text { SDSS13Y00031T-0B- } \\
\text { 0Y-0M-0Y-0B-44Y }\end{array}$ & 9.17 & 4.62 & 1.89 & $\mathrm{~S}$ \\
\hline 153 & 620648 & $\begin{array}{l}\text { T.DICOCCON PI94625/AE.SQUARROSA } \\
\text { (372)//COPIO/3/KACHU \#1/KIRITATI//KACHU }\end{array}$ & $\begin{array}{l}\text { SDSS13Y00079T-0B- } \\
\text { 0Y-0M-0Y-0B-112Y }\end{array}$ & 9.17 & 5.71 & 3.00 & S \\
\hline 177 & 620657 & $\begin{array}{l}\text { CETA/AE.SQUARROSA (895)/3/KACHU \#1/ } \\
\text { KIRITATI//KACHU/4/PBW343*2/KUKUNA*2//FRTL/ } \\
\text { PIFED }\end{array}$ & $\begin{array}{l}\text { SDSS13Y00088T-0B- } \\
\text { 0Y-0M-0Y-0B-115Y }\end{array}$ & 9.17 & 4.96 & 2.02 & $S$ \\
\hline 266 & 620759 & $\begin{array}{l}\text { PERSIA-88/3/PBW343*2/KUKUNA*2//FRTL/PIFED } \\
\text { /4/QUAIU \#1 }\end{array}$ & $\begin{array}{l}\text { SDSS13Y00190T-0B- } \\
\text { 0Y-0M-0Y-0B-89Y }\end{array}$ & 9.17 & 4.45 & 1.82 & $\mathrm{~S}$ \\
\hline $\begin{array}{l}\text { KUTLUK } \\
\text { (S) }\end{array}$ & & $\begin{array}{l}\text { KRASNODARSKAYA//INIA-66/LILIFEN/3/ } \\
\text { CALIBASAN }\end{array}$ & & 9.25 & 8.62 & 4.31 & S \\
\hline 12 & 620577 & DVERD_2/AE.SQUARROSA (333)//BAJ \#1/3/SUP152 & $\begin{array}{l}\text { SDSS13Y00008T-0B- } \\
\text { 0Y-0M-0Y-0B-64Y }\end{array}$ & 9.33 & 5.79 & 2.36 & $\mathrm{~S}$ \\
\hline 19 & 620583 & H-1624/BAJ \#1//SUP152 & $\begin{array}{l}\text { SDSS13Y00014T-0B- } \\
\text { 0Y-0M-0Y-0B-31Y }\end{array}$ & 9.33 & 3.44 & 2.75 & $\mathrm{~S}$ \\
\hline 193 & 620670 & $\begin{array}{l}\text { LOCAL RED/AE.SQUARROSA (222)/3/PBW343*2/ } \\
\text { KUKUNA*2//FRTL/PIFED/4/QUAIU \#1 }\end{array}$ & $\begin{array}{l}\text { SDSS13Y00101T-0B- } \\
\text { 0Y-0M-0Y-0B-81Y }\end{array}$ & 9.33 & 4.68 & 1.91 & S \\
\hline 141 & 620646 & IWA8611400/BORL14//COPIO & $\begin{array}{l}\text { SDSS13Y00077T-0B- } \\
\text { 0Y-0M-0Y-0B-92Y }\end{array}$ & 9.50 & 5.13 & 2.09 & $\mathrm{~S}$ \\
\hline $\begin{array}{l}\text { KUTLUK } \\
\quad \text { (S) }\end{array}$ & & $\begin{array}{l}\text { KRASNODARSKAYA//INIA- } \\
\text { 66/LILIFEN/3/CALIBASAN }\end{array}$ & & 9.50 & 4.73 & 2.36 & $\mathrm{~S}$ \\
\hline 147 & 620647 & $\begin{array}{l}\text { T.DICOCCON PI94624/AE.SQUARROSA (454)// } \\
\text { COPIO/3/KACHU \#1/KIRITATI//KACHU }\end{array}$ & $\begin{array}{l}\text { SDSS13Y00078T-0B- } \\
\text { 0Y-0M-0Y-0B-86Y }\end{array}$ & 9.67 & 6.71 & 2.74 & $\mathrm{~S}$ \\
\hline 190 & 620667 & $\begin{array}{l}\text { DOY1/AE.SQUARROSA (415)/3/PBW343*2/ } \\
\text { KUKUNA*2//FRTL/PIFED/4/QUAIU \#1 }\end{array}$ & $\begin{array}{l}\text { SDSS13Y00098T-0B- } \\
\text { 0Y-0M-0Y-0B-69Y }\end{array}$ & 9.67 & 5.24 & 2.14 & $\mathrm{~S}$ \\
\hline 233 & 620695 & INDIA-107/KACHU//BAJ \#1 & $\begin{array}{l}\text { SDSS13Y00126T-0B- } \\
\text { 0Y-0M-0Y-0B-103Y }\end{array}$ & 9.67 & 6.65 & 2.72 & $\mathrm{~S}$ \\
\hline 13 & 620577 & DVERD_2/AE.SQUARROSA (333)//BAJ \#1/3/SUP152 & $\begin{array}{l}\text { SDSS13Y00008T-0B- } \\
\text { 0Y-0M-0Y-0B-65Y }\end{array}$ & 9.83 & 6.52 & 3.50 & $\mathrm{~S}$ \\
\hline 25 & 620586 & $\begin{array}{l}\text { INDIA-101/3/FRET2*2/SHAMA//KACHU/4/ } \\
\text { HUW234+LR34/PRINIA*2//KIRITATI }\end{array}$ & $\begin{array}{l}\text { SDSS13Y00017T-0B- } \\
\text { 0Y-0M-0Y-0B-43Y }\end{array}$ & 9.83 & 6.62 & 3.11 & $\mathrm{~S}$ \\
\hline 46 & 620600 & $\begin{array}{l}\text { H-1357/8/CNDO/R143//ENTE/MEXI_2/3/AEGILOPS } \\
\text { SQUARROSA (TAUS)/4/WEAVER/5/PICUS/6/ } \\
\text { TROST/7/TACUPETO F2001/9/KAUZ//ALTAR 84/ } \\
\text { AOS/3/PASTOR/4/MILAN/CUPE//SW89.3064/5/KIRIT } \\
\text { ATI }\end{array}$ & $\begin{array}{l}\text { SDSS13Y00031T-0B- } \\
\text { 0Y-0M-0Y-0B-51Y }\end{array}$ & 9.83 & 5.49 & 2.24 & $\mathrm{~S}$ \\
\hline 186 & 620662 & $\begin{array}{l}\text { YAV79//DACK/RABI/3/SNIPE/4/AE.SQUARROSA } \\
(477) / 5 / \mathrm{KACHU} \# 1 / \mathrm{KIRITATI//KACHU/6/PBW343*2/} \\
\text { KUKUNA*2//FRTL/PIFED }\end{array}$ & $\begin{array}{l}\text { SDSS13Y00093T-0B- } \\
\text { 0Y-0M-0Y-0B-97Y }\end{array}$ & 9.83 & 6.62 & 2.70 & $\mathrm{~S}$ \\
\hline 198 & 620678 & H-1491/ROLF07//NAVJ07 & $\begin{array}{l}\text { SDSS13Y00109T-0B- } \\
\text { 0Y-0M-0Y-0B-74Y }\end{array}$ & 9.83 & 4.54 & 1.85 & $\mathrm{~S}$ \\
\hline 235 & 620696 & $\begin{array}{l}\text { IG 41242/3/PBW343*2/KUKUNA*2//FRTL/ } \\
\text { PIFED/4/QUAIU \#1 }\end{array}$ & $\begin{array}{l}\text { SDSS13Y00127T-0B- } \\
\text { 0Y-0M-0Y-0B-75Y }\end{array}$ & 9.83 & 6.08 & 2.48 & $\mathrm{~S}$ \\
\hline 5 & 620577 & DVERD_2/AE.SQUARROSA (333)//BAJ \#1/3/SUP152 & $\begin{array}{l}\text { SDSS13Y00008T-0B- } \\
\text { 0Y-0M-0Y-0B-47Y }\end{array}$ & 10.00 & 5.18 & 3.04 & $\mathrm{~S}$ \\
\hline 72 & 620629 & H-1546/NELOKI/3/ATTILA*2/PBW65//MURGA & $\begin{array}{l}\text { SDSS13Y00060T-0B- } \\
\text { 0Y-0M-0Y-0B-44Y }\end{array}$ & 10.00 & 6.07 & 3.31 & $\mathrm{~S}$ \\
\hline 105 & 620638 & $\begin{array}{l}\text { LOCAL RED/AE. SQUARROSA (223)//BORL14/3/ } \\
\text { COPIO }\end{array}$ & $\begin{array}{l}\text { SDSS13Y00069T-0B- } \\
\text { 0Y-0M-0Y-0B-39Y }\end{array}$ & 10.00 & 10.18 & 4.16 & $\mathrm{~S}$ \\
\hline 230 & 620694 & INDIA-59/KACHU//BAJ \#1 & $\begin{array}{l}\text { SDSS13Y00125T-0B- } \\
\text { 0Y-0M-0Y-0B-91Y }\end{array}$ & 10.00 & 6.72 & 3.59 & $\mathrm{~S}$ \\
\hline $\begin{array}{l}\text { BEZOST } \\
\text { AJA (S) }\end{array}$ & & LUT17/SRS2 & & 10.00 & 4.24 & 2.12 & $\mathrm{~S}$ \\
\hline 14 & 620579 & $\begin{array}{l}\text { D67.2/PARANA 66.270//AE.SQUARROSA } \\
\text { (677)/3/BAJ \#1/4/SUP152 }\end{array}$ & $\begin{array}{l}\text { SDSS13Y00010T-0B- } \\
\text { 0Y-0M-0Y-0B-35Y }\end{array}$ & 10.17 & 5.95 & 3.44 & HS \\
\hline 79 & 620630 & H-1694/NELOKI/3/ATTILA*2/PBW65//MURGA & $\begin{array}{l}\text { SDSS13Y00061T-0B- } \\
\text { 0Y-0M-0Y-0B-49Y }\end{array}$ & 10.17 & 3.97 & 1.62 & HS \\
\hline 103 & 620637 & TXL92.8.1/3/ATTILA*2/PBW65//MURGA/4/BORL14 & $\begin{array}{l}\text { SDSS13Y00068T-0B- } \\
\text { 0Y-0M-0Y-0B-65Y }\end{array}$ & 10.17 & 5.64 & 2.91 & HS \\
\hline 258 & 620746 & $\begin{array}{l}\text { IG 122738/3/PBW343*2/KUKUNA*2// } \\
\text { FRTL/PIFED/4/QUAIU \#1 }\end{array}$ & $\begin{array}{l}\text { SDSS13Y00177T-0B- } \\
\text { 0Y-0M-0Y-0B-59Y }\end{array}$ & 10.17 & 6.68 & 3.09 & HS \\
\hline 92 & 620634 & INDIA-38/3/ATTILA*2/PBW65//MURGA/4/BORL14 & $\begin{array}{l}\text { SDSS13Y00065T-0B- } \\
\text { 0Y-0M-0Y-0B-54Y }\end{array}$ & 10.33 & 6.53 & 3.02 & HS \\
\hline 172 & 620654 & $\begin{array}{l}\text { CETA/AE.SQUARROSA ( } 850) / 3 / \text { KACHU } \# 1 / \\
\text { KIRITATI//KACHU/4/PBW343*2/KUKUNA*2//FRTL/ } \\
\text { PIFED }\end{array}$ & $\begin{array}{l}\text { SDSS13Y00085T-0B- } \\
\text { 0Y-0M-0Y-0B-64Y }\end{array}$ & 10.33 & 4.23 & 1.73 & HS \\
\hline 188 & 620665 & $\begin{array}{l}\text { GARZA/BOY//AE.SQUARROSA (281)/3/PBW343*2/ } \\
\text { KUKUNA*2//FRTL/PIFED/4/QUAIU \#1 }\end{array}$ & $\begin{array}{l}\text { SDSS13Y00096T-0B- } \\
\text { 0Y-0M-0Y-0B-58Y }\end{array}$ & 10.33 & 5.54 & 2.26 & HS \\
\hline
\end{tabular}


Supplementary Table8(Continue): List of Drought and Heat Tolerant Spring Wheat lines and their Resistant Response to the CCN Heterodera filipjevi. (SD) Standard Deviation, (SE) Standard Error, (CID) Cross ID

\begin{tabular}{|c|c|c|c|c|c|c|c|}
\hline $\begin{array}{c}\text { ENTRY } \\
\text { NUMBER }\end{array}$ & $C I D$ & PEDIGREE (CROSS) & $\begin{array}{l}\text { SELECTION } \\
\text { HISTORY }\end{array}$ & $\begin{array}{c}\text { (MEAN) } \\
\text { CYSTS } \\
\text { AND } \\
\text { FEMALES }\end{array}$ & $S D$ & $S E$ & $\begin{array}{l}\text { RESISTANT } \\
\text { RESPONSE }\end{array}$ \\
\hline 192 & 620669 & $\begin{array}{l}\text { LOCAL RED/AE.SQUARROSA (220)/3/PBW343*2/ } \\
\text { KUKUNA*2//FRTL/PIFED/4/QUAIU \#1 }\end{array}$ & $\begin{array}{l}\text { SDSS13Y00100T-0B- } \\
\text { 0Y-0M-0Y-0B-87Y }\end{array}$ & 10.33 & 6.44 & 3.36 & HS \\
\hline 21 & 620583 & H-1624/BAJ \#1//SUP152 & $\begin{array}{l}\text { SDSS13Y00014T-0B- } \\
\text { 0Y-0M-0Y-0B-34Y }\end{array}$ & 10.50 & 5.89 & 2.40 & HS \\
\hline 120 & 620641 & $\begin{array}{l}\text { 68.111/RGB-U//WARD } \\
\text { RESEL/3/STIL/4/AE.SQUARROSA } \\
\text { (630)/5/BORL14/6/COPIO }\end{array}$ & $\begin{array}{l}\text { SDSS13Y00072T-0B- } \\
\text { 0Y-0M-0Y-0B-45Y }\end{array}$ & 10.50 & 3.15 & 1.28 & HS \\
\hline 122 & 620641 & $\begin{array}{l}\text { 68.111/RGB-U//WARD } \\
\text { RESEL/3/STIL/4/AE.SQUARROSA } \\
\text { (630)/5/BORL14/6/COPIO }\end{array}$ & $\begin{array}{l}\text { SDSS13Y00072T-0B- } \\
\text { 0Y-0M-0Y-0B-48Y }\end{array}$ & 10.50 & 5.24 & 3.25 & HS \\
\hline 3 & 620575 & DOY1/AE.SQUARROSA (488)//BAJ \#1/3/SUP152 & $\begin{array}{l}\text { SDSS13Y00006T-0B- } \\
\text { 0Y-0M-0Y-0B-56Y }\end{array}$ & 10.67 & 6.74 & 2.75 & HS \\
\hline 65 & 620625 & $\begin{array}{l}\text { IG } 122795 / 4 / \mathrm{PRL} / 2 * \mathrm{PASTOR} / / \\
\text { PBW343*2/KUKUNA/3/ROLF07/5/NELOKI }\end{array}$ & $\begin{array}{l}\text { SDSS13Y00056T-0B- } \\
\text { 0Y-0M-0Y-0B-96Y }\end{array}$ & 10.67 & 6.31 & 2.58 & HS \\
\hline 95 & 620634 & INDIA-38/3/ATTILA*2/PBW65//MURGA/4/BORL14 & $\begin{array}{l}\text { SDSS13Y00065T-0B- } \\
\text { 0Y-0M-0Y-0B-60Y }\end{array}$ & 10.67 & 6.65 & 2.72 & HS \\
\hline 125 & 620641 & $\begin{array}{l}\text { 68.111/RGB-U//WARD RESEL/3/STIL/4/ } \\
\text { AE.SQUARROSA (630)/5/BORL14/6/COPIO }\end{array}$ & $\begin{array}{l}\text { SDSS13Y00072T-0B- } \\
\text { 0Y-0M-0Y-0B-52Y }\end{array}$ & 10.67 & 6.98 & 2.85 & HS \\
\hline 175 & 620655 & $\begin{array}{l}\text { CETA/AE.SQUARROSA }(872) / 3 / \text { KACHU } \# 1 / \\
\text { KIRITATI//KACHU/4/PBW343*2/KUKUNA*2//FRTL/ } \\
\text { PIFED }\end{array}$ & $\begin{array}{l}\text { SDSS13Y00086T-0B- } \\
\text { 0Y-0M-0Y-0B-64Y }\end{array}$ & 10.67 & 6.77 & 3.13 & HS \\
\hline 35 & 620594 & $\begin{array}{l}\text { INDIA-223/7/SHA7/VEE\#5/5/VEE\#8//JUP/BJY/3/ } \\
\text { F3.71/TRM/4/2*WEAVER/6/SKAUZ/PARUS//PARUS/ } \\
\text { 8/CNDO/R143//ENTE/MEXI_2/3/AEGILOPS } \\
\text { SQUARROSA (TAUS)/4/WEAVER/5/PICUS/6/ } \\
\text { TROST/7/TACUPETO F2001 }\end{array}$ & $\begin{array}{l}\text { SDSS13Y00025T-0B- } \\
\text { 0Y-0M-0Y-0B-36Y }\end{array}$ & 10.83 & 2.04 & 0.83 & HS \\
\hline 50 & 620611 & $\begin{array}{l}\text { CETA/AE.SQUARROSA (391)/4/PRL/2*PASTOR// } \\
\text { PBW343*2/KUKUNA/3/ROLF07/5/NELOKI }\end{array}$ & $\begin{array}{l}\text { SDSS13Y00042T-0B- } \\
\text { 0Y-0M-0Y-0B-6Y }\end{array}$ & 10.83 & 6.15 & 3.89 & HS \\
\hline 63 & 620621 & $\begin{array}{l}\text { IG 122196/4/PRL/2*PASTOR//PBW343*2/ } \\
\text { KUKUNA/3/ROLF07/5/NELOKI }\end{array}$ & $\begin{array}{l}\text { SDSS13Y00052T-0B- } \\
\text { 0Y-0M-0Y-0B-80Y }\end{array}$ & 10.83 & 6.62 & 2.70 & HS \\
\hline 180 & 620661 & 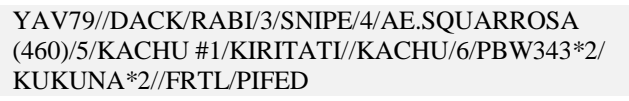 & $\begin{array}{l}\text { SDSS13Y00092T-0B- } \\
\text { 0Y-0M-0Y-0B-97Y }\end{array}$ & 10.83 & 6.62 & 2.70 & HS \\
\hline 191 & 620669 & $\begin{array}{l}\text { LOCAL RED/AE.SQUARROSA (220)/3/PBW343*2/ } \\
\text { KUKUNA*2//FRTL/PIFED/4/QUAIU \#1 }\end{array}$ & $\begin{array}{l}\text { SDSS13Y00100T-0B- } \\
\text { 0Y-0M-0Y-0B-79Y }\end{array}$ & 11.00 & 7.40 & 3.74 & HS \\
\hline 111 & 620638 & $\begin{array}{l}\text { LOCAL RED/AE.SQUARROSA (223)//BORL14/3/ } \\
\text { COPIO }\end{array}$ & $\begin{array}{l}\text { SDSS13Y00069T-0B- } \\
\text { 0Y-0M-0Y-0B-65Y }\end{array}$ & 11.17 & 6.40 & 3.29 & HS \\
\hline 176 & 620657 & $\begin{array}{l}\text { CETA/AE.SQUARROSA ( } 895) / 3 / \mathrm{KACHU} \# 1 / \\
\text { KIRITATI//KACHU/4/PBW343*2/KUKUNA*2//FRTL/ } \\
\text { PIFED }\end{array}$ & $\begin{array}{l}\text { SDSS13Y00088T-0B- } \\
\text { 0Y-0M-0Y-0B-110Y }\end{array}$ & 11.17 & 7.00 & 2.86 & HS \\
\hline 71 & 620629 & H-1546/NELOKI/3/ATTILA*2/PBW65//MURGA & $\begin{array}{l}\text { SDSS13Y00060T-0B- } \\
\text { 0Y-0M-0Y-0B-33Y }\end{array}$ & 11.33 & 7.09 & 3.38 & HS \\
\hline 138 & 620645 & IWA8612416/BORL14//COPIO & $\begin{array}{l}\text { SDSS13Y00076T-0B- } \\
\text { 0Y-0M-0Y-0B-48Y }\end{array}$ & 11.33 & 6.56 & 2.68 & HS \\
\hline 173 & 620654 & $\begin{array}{l}\text { CETA/AE.SQUARROSA }(850) / 3 / \text { KACHU } \# 1 / \\
\text { KIRITATI/KACHU/4/PBW343*2/KUKUNA*2//FRTL/ } \\
\text { PIFED }\end{array}$ & $\begin{array}{l}\text { SDSS13Y00085T-0B- } \\
\text { 0Y-0M-0Y-0B-69Y }\end{array}$ & 11.33 & 7.39 & 3.90 & HS \\
\hline 232 & 620695 & INDIA-107/KACHU//BAJ \#1 & $\begin{array}{l}\text { SDSS13Y00126T-0B- } \\
\text { 0Y-0M-0Y-0B-101Y }\end{array}$ & 11.33 & 7.39 & 3.02 & HS \\
\hline 94 & 620634 & INDIA-38/3/ATTILA*2/PBW65//MURGA/4/BORL14 & $\begin{array}{l}\text { SDSS13Y00065T-0B- } \\
\text { 0Y-0M-0Y-0B-58Y }\end{array}$ & 11.50 & 7.87 & 4.01 & HS \\
\hline 107 & 620638 & $\begin{array}{l}\text { LOCAL RED/AE.SQUARROSA (223)//BORL14/3/ } \\
\text { COPIO }\end{array}$ & $\begin{array}{l}\text { SDSS13Y00069T-0B- } \\
\text { 0Y-0M-0Y-0B-52Y }\end{array}$ & 11.50 & 7.29 & 2.97 & HS \\
\hline 117 & 620640 & $\begin{array}{l}\text { 68.111/RGB-U//WARD RESEL/3/STIL/4/ } \\
\text { AE.SQUARROSA (628)/5/BORL14/6/COPIO }\end{array}$ & $\begin{array}{l}\text { SDSS13Y00071T-0B- } \\
\text { 0Y-0M-0Y-0B-53Y }\end{array}$ & 11.50 & 5.92 & 3.50 & HS \\
\hline 96 & 620635 & INDIA-50/3/ATTILA*2/PBW65//MURGA/4/BORL14 & $\begin{array}{l}\text { SDSS13Y00066T-0B- } \\
\text { 0Y-0M-0Y-0B-59Y }\end{array}$ & 11.67 & 7.31 & 3.38 & HS \\
\hline 140 & 620645 & IWA8612416/BORL14//COPIO & $\begin{array}{l}\text { SDSS13Y00076T-0B- } \\
\text { 0Y-0M-0Y-0B-50Y }\end{array}$ & 11.67 & 8.02 & 3.27 & HS \\
\hline 6 & 620577 & DVERD_2/AE.SQUARROSA (333)//BAJ \#1/3/SUP152 & $\begin{array}{l}\text { SDSS13Y00008T-0B- } \\
\text { 0Y-0M-0Y-0B-51Y }\end{array}$ & 11.83 & 8.04 & 3.28 & HS \\
\hline 26 & 620586 & $\begin{array}{l}\text { INDIA- } \\
\text { 101/3/FRET2*2/SHAMA//KACHU/4/HUW234+LR34/P } \\
\text { RINIA*2//KIRITATI }\end{array}$ & $\begin{array}{l}\text { SDSS13Y00017T-0B- } \\
\text { 0Y-0M-0Y-0B-54Y }\end{array}$ & 11.83 & 6.37 & 2.60 & HS \\
\hline 66 & 620627 & $\begin{array}{l}\text { 68.111/RGB-U//WARD/3/FGO/4/RABI/5/ } \\
\text { AE.SQUARROSA (890)/6/NELOKI/7/ATTILA*2/ } \\
\text { PBW65//MURGA }\end{array}$ & $\begin{array}{l}\text { SDSS13Y00058T-0B- } \\
\text { 0Y-0M-0Y-0B-43Y }\end{array}$ & 11.83 & 6.62 & 3.48 & HS \\
\hline 80 & 620630 & H-1694/NELOKI/3/ATTILA*2/PBW65//MURGA & $\begin{array}{l}\text { SDSS13Y00061T-0B- } \\
\text { 0Y-0M-0Y-0B-55Y }\end{array}$ & 11.83 & 6.65 & 2.71 & HS \\
\hline 121 & 620641 & $\begin{array}{l}\text { 68.111/RGB-U//WARD RESEL/3/STIL/4/ } \\
\text { AE.SQUARROSA (630)/5/BORL14/6/COPIO }\end{array}$ & $\begin{array}{l}\text { SDSS13Y00072T-0B- } \\
\text { 0Y-0M-0Y-0B-47Y }\end{array}$ & 12.00 & 8.02 & 3.28 & HS \\
\hline
\end{tabular}


Supplementary Table9(Continue): List of Drought and Heat Tolerant Spring Wheat lines and their Resistant Response to the CCN Heterodera filipjevi. (SD) Standard Deviation, (SE) Standard Error, (CID) Cross ID

\begin{tabular}{|c|c|c|c|c|c|c|c|}
\hline $\begin{array}{l}\text { ENTRY } \\
\text { NUMBER }\end{array}$ & $C I D$ & PEDIGREE (CROSS) & $\begin{array}{l}\text { SELECTION } \\
\text { HISTORY }\end{array}$ & $\begin{array}{c}\text { (MEAN) } \\
\text { CYSTS } \\
\text { AND } \\
\text { FEMALES }\end{array}$ & $S D$ & $S E$ & $\begin{array}{l}\text { RESISTANT } \\
\text { RESPONSE }\end{array}$ \\
\hline 132 & 620642 & $\begin{array}{l}\text { D67.2/PARANA 66.270//AE.SQUARROSA (1085)/3/ } \\
\text { BORL14/4/COPIO }\end{array}$ & $\begin{array}{l}\text { SDSS13Y00073T-0B- } \\
\text { 0Y-0M-0Y-0B-77Y }\end{array}$ & 12.00 & 7.51 & 3.07 & HS \\
\hline 185 & 620662 & $\begin{array}{l}\text { YAV79//DACK/RABI/3/SNIPE/4/AE.SQUARROSA } \\
\text { (477)/5/KACHU \#1/KIRITATI//KACHU/6/ } \\
\text { PBW343*2/KUKUNA*2//FRTL/PIFED }\end{array}$ & $\begin{array}{l}\text { SDSS13Y00093T-0B- } \\
\text { 0Y-0M-0Y-0B-91Y }\end{array}$ & 12.00 & 4.82 & 1.97 & HS \\
\hline 156 & 620650 & IG 41620/COPIO/3/KACHU \#1/KIRITATI//KACHU & $\begin{array}{l}\text { SDSS13Y00081T-0B- } \\
\text { 0Y-0M-0Y-0B-78Y }\end{array}$ & 12.17 & 5.12 & 2.09 & HS \\
\hline 260 & 620747 & $\begin{array}{l}\text { IG 122740/3/PBW343*2/KUKUNA*2//FRTL/ } \\
\text { PIFED/4/QUAIU \#1 }\end{array}$ & $\begin{array}{l}\text { SDSS13Y00178T-0B- } \\
\text { 0Y-0M-0Y-0B-47Y }\end{array}$ & 12.33 & 8.12 & 3.87 & HS \\
\hline 152 & 620648 & $\begin{array}{l}\text { T.DICOCCON PI94625/AE.SQUARROSA (372)// } \\
\text { COPIO/3/KACHU \#1/KIRITATI//KACHU }\end{array}$ & $\begin{array}{l}\text { SDSS13Y00079T-0B- } \\
\text { 0Y-0M-0Y-0B-108Y }\end{array}$ & 12.50 & 6.12 & 3.59 & HS \\
\hline 116 & 620640 & $\begin{array}{l}\text { 68.111/RGB-U//WARD RESEL/3/STIL } \\
\text { /4/AE.SQUARROSA (628)/5/BORL14/6/COPIO }\end{array}$ & $\begin{array}{l}\text { SDSS13Y00071T-0B- } \\
\text { 0Y-0M-0Y-0B-51Y }\end{array}$ & 12.67 & 7.81 & 4.82 & HS \\
\hline 133 & 620642 & $\begin{array}{l}\text { D67.2/PARANA 66.270//AE.SQUARROSA (1085)/3/ } \\
\text { BORL14/4/COPIO }\end{array}$ & $\begin{array}{l}\text { SDSS13Y00073T-0B- } \\
\text { 0Y-0M-0Y-0B-79Y }\end{array}$ & 12.67 & 7.26 & 2.96 & HS \\
\hline 161 & 61665 & EMPTY PLOT & & 12.67 & 8.04 & 3.28 & HS \\
\hline 213 & 620687 & CROC_1/AE.SQUARROSA (436)//KACHU/3/BAJ \#1 & $\begin{array}{l}\text { SDSS13Y00118T-0B- } \\
\text { 0Y-0M-0Y-0B-129Y }\end{array}$ & 12.67 & 7.84 & 3.20 & HS \\
\hline 85 & 620633 & IG 131672/3/ATTILA*2/PBW65//MURGA/4/BORL14 & $\begin{array}{l}\text { SDSS13Y00064T-0B- } \\
\text { 0Y-0M-0Y-0B-50Y }\end{array}$ & 13.17 & 6.37 & 3.79 & HS \\
\hline 104 & 620637 & TXL92.8.1/3/ATTILA*2/PBW65//MURGA/4/BORL14 & $\begin{array}{l}\text { SDSS13Y00068T-0B- } \\
\text { 0Y-0M-0Y-0B-67Y }\end{array}$ & 13.17 & 5.64 & 2.30 & HS \\
\hline 151 & 620648 & $\begin{array}{l}\text { T.DICOCCON PI94625/AE.SQUARROSA (372)// } \\
\text { COPIO/3/KACHU \#1/KIRITATI//KACHU }\end{array}$ & $\begin{array}{l}\text { SDSS13Y00079T-0B- } \\
\text { 0Y-0M-0Y-0B-107Y }\end{array}$ & 13.17 & 7.76 & 3.99 & HS \\
\hline 194 & 620674 & JAL95.4.3/VORB//ROLF07 & $\begin{array}{l}\text { SDSS13Y00105T-0B- } \\
\text { 0Y-0M-0Y-0B-29Y }\end{array}$ & 13.33 & 9.14 & 4.91 & HS \\
\hline 15 & 620579 & $\begin{array}{l}\text { D67.2/PARANA 66.270//AE.SQUARROSA } \\
\text { (677)/3/BAJ \#1/4/SUP152 }\end{array}$ & $\begin{array}{l}\text { SDSS13Y00010T-0B- } \\
\text { 0Y-0M-0Y-0B-47Y }\end{array}$ & 13.50 & 8.31 & 3.39 & HS \\
\hline 89 & 620634 & INDIA-38/3/ATTILA*2/PBW65//MURGA/4/BORL14 & $\begin{array}{l}\text { SDSS13Y00065T-0B- } \\
\text { 0Y-0M-0Y-0B-46Y }\end{array}$ & 13.50 & 9.31 & 4.88 & HS \\
\hline 187 & 620663 & $\begin{array}{l}\text { GARZA/BOY//AE.SQUARROSA (278)/3/PBW343*2/ } \\
\text { KUKUNA*2//FRTL/PIFED/4/QUAIU \#1 }\end{array}$ & $\begin{array}{l}\text { SDSS13Y00094T-0B- } \\
\text { 0Y-0M-0Y-0B-47Y }\end{array}$ & 13.50 & 7.69 & 4.06 & HS \\
\hline 126 & 620641 & $\begin{array}{l}\text { 68.111/RGB-U//WARD RESEL/3/STIL/4/ } \\
\text { AE.SQUARROSA (630)/5/BORL14/6/COPIO }\end{array}$ & $\begin{array}{l}\text { SDSS13Y00072T-0B- } \\
\text { 0Y-0M-0Y-0B-56Y }\end{array}$ & 13.67 & 8.26 & 3.37 & HS \\
\hline 101 & 620635 & INDIA-50/3/ATTILA*2/PBW65//MURGA/4/BORL14 & $\begin{array}{l}\text { SDSS13Y00066T-0B- } \\
\text { 0Y-0M-0Y-0B-66Y }\end{array}$ & 14.00 & 8.37 & 3.42 & HS \\
\hline 100 & 620635 & INDIA-50/3/ATTILA*2/PBW65//MURGA/4/BORL14 & $\begin{array}{l}\text { SDSS13Y00066T-0B- } \\
\text { 0Y-0M-0Y-0B-63Y }\end{array}$ & 15.00 & 8.49 & 3.46 & HS \\
\hline 7 & 620577 & DVERD_2/AE.SQUARROSA (333)//BAJ \#1/3/SUP152 & $\begin{array}{l}\text { SDSS13Y00008T-0B- } \\
\text { 0Y-0M-0Y-0B-53Y }\end{array}$ & 15.50 & 8.34 & 3.40 & HS \\
\hline 91 & 620634 & INDIA-38/3/ATTILA*2/PBW65//MURGA/4/BORL14 & $\begin{array}{l}\text { SDSS13Y00065T-0B- } \\
\text { 0Y-0M-0Y-0B-53Y }\end{array}$ & 15.67 & 10.75 & 6.02 & HS \\
\hline 8 & 620577 & DVERD_2/AE.SQUARROSA (333)//BAJ \#1/3/SUP152 & $\begin{array}{l}\text { SDSS13Y00008T-0B- } \\
\text { 0Y-0M-0Y-0B-54Y }\end{array}$ & 17.50 & 10.01 & 5.15 & HS \\
\hline
\end{tabular}

(C) 2022 by the author(s). Published by Ankara University, Faculty of Agriculture, Ankara, Turkey. This is an Open Access article distributed under the terms and conditions of the Creative Commons Attribution (CC BY) license (http://creativecommons.org/licenses/by/4.0/), which permits unrestricted use, distribution, and reproduction in any medium, provided the original work is properly cited. 